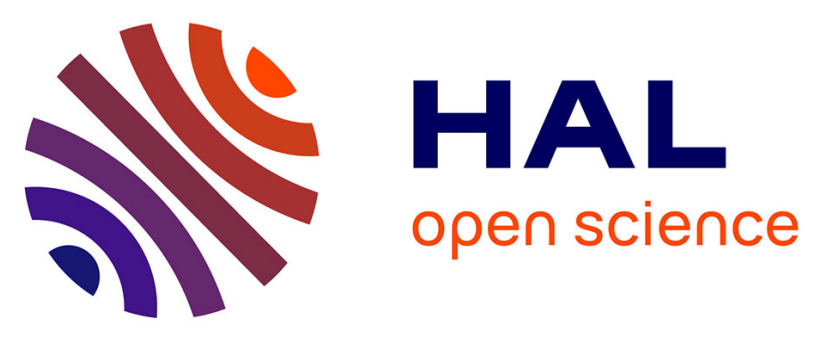

\title{
Augmented Lagrangian and penalty methods for the simulation of two-phase flows interacting with moving solids. Application to hydroplaning flows interacting with real tire tread patterns
}

Vincent Stéphane, Arthur Sarthou, Jean-Paul Caltagirone, Sonilhac Fabien, Février Pierre, Mignot Christian, Grégoire Pianet

\section{To cite this version:}

Vincent Stéphane, Arthur Sarthou, Jean-Paul Caltagirone, Sonilhac Fabien, Février Pierre, et al.. Augmented Lagrangian and penalty methods for the simulation of two-phase flows interacting with moving solids. Application to hydroplaning flows interacting with real tire tread patterns. Journal of Computational Physics, 2011, 230 (4), pp.956-983. 10.1016/j.jcp.2010.10.006 . hal-00424018v2

\author{
HAL Id: hal-00424018 \\ https://hal.science/hal-00424018v2
}

Submitted on 6 Sep 2010

HAL is a multi-disciplinary open access archive for the deposit and dissemination of scientific research documents, whether they are published or not. The documents may come from teaching and research institutions in France or abroad, or from public or private research centers.
L'archive ouverte pluridisciplinaire HAL, est destinée au dépôt et à la diffusion de documents scientifiques de niveau recherche, publiés ou non, émanant des établissements d'enseignement et de recherche français ou étrangers, des laboratoires publics ou privés. 


\title{
Augmented Lagrangian and penalty methods for the simulation of two-phase flows interacting with moving solids. Application to hydroplaning flows interacting with real tire tread patterns
}

\author{
Stéphane Vincent*, Arthur Sarthou*, Jean-Paul Caltagirone*, Fabien Sonilhac*, \\ Pierre Février*, Christian Mignot*, Grégoire Pianet *, \\ vincent@enscpb.fr (corresponding author), Tel.: (+33) 540002707
}

\$. Université de Bordeaux, Transfert, Ecoulements, Fluides, Energétique TREFLE, UMR CNRS 8508, 16 Avenue Pey-Berland, $3360^{77}$ PESSAC Cedex, France

* La manufacture des pneumatiques MICHELIN,

23, Place des Carmes-Déchaux, 63040 CLERMONT-FERRAND Cedex 9, France

\begin{abstract}
The numerical simulation of the interaction between a free surface flow and a moving obstacle is considered for the analysis of hydroplaning flows. A new augmented Lagrangian method, coupled to fictitious domains and penalty methods, is proposed for the simulation of multiphase flows. The augmented Lagrangian parameter is estimated by an automatic analysis of the discretization matrix resulting from the approximation of the momentum equations. The algebraic automatic augmented Lagrangian 3AL approach is validated on the natural convection in a differentially heated cavity, a two-dimensional collapse of a water column, the three-dimensional settling of a particle in a tank and the falling of a dense cylinder in air. Finally, the 3AL method is utilized to simulate the hydroplaning of a tire under various pattern shape conditions.

key-words

fictitious domain, augmented Lagrangian, 1-fluid model, penalty method, Volume Of Fluid,
\end{abstract}


patterned tire, hydroplaning flows 


\section{Introduction}

The present article aims at providing a fictitious domain model for dealing with two-phase flows interacting with moving obstacles. The objective is to simulate hydroplaning phenomena with the proposed model and numerical methods and to characterize the efforts exerted by the free surface flow on a tire.

The hydroplaning is a phenomenon resulting from the loss of contact between a tire and the road when a vehicle is moving at a certain speed on a wet road. For a given velocity, the interaction between the water laying on the road and the tire generates a water reserve in front of the tire which is higher than the initial water depth. A resulting pressure is generated at the tire surface around the contact area between the tire and the water reserve. When the vertical effort generated by this pressure becomes superior to the weight of the vehicle, the contact between this vehicle and the road is no more maintained and the hydroplaning occurs: the adherence between the vehicle and the road is lost and the trajectory of the vehicle is no more controlled. Hydroplaning is responsible for the major wet road performances of vehicles such as the wet road braking and the driving controllability. From the literature, it is well known that the link between the vehicle velocity and the hydroplaning pressure follows at first order [27]

$$
P_{h}=K V_{v}^{2}
$$

where $P_{h}$ is the hydroplaning pressure, $V_{v}$ is the velocity of the vehicle and $K$ is a constant equal to half the density of water. This law is obtained under Bernouilli's assumptions of perfect fluid behavior. From the tire manufacturer experience, it is well known that the more efficient way to decrease $P_{h}$ for given wetting conditions of road is to incorporate specific tread patterns. In this way, more incoming water is evacuated laterally, more water reserve generated in front of the tire is reduced for a given velocity and then the hydroplaning effect appears for higher vehicle velocities. This effect of the tire tread pattern has been studied 
for example by Masataka and Toshihiko [23].

Few existing experimental or theoretical studies have been able to predict quantitatively the improvement brought by the choice of a tire structure on the hydroplaning compared to a reference flat tire whereas building a tire is very expensive. The aim of the present article is to propose a modeling methodology dedicated to the numerical simulation of two-phase flows interacting with solids and in particular to the prediction of hydroplaning effects and classification of tire structures. This objective requires to account for three-dimensional turbulent free surface air-water flows interacting with complex tire geometries.

Among the rare existing literature works in the field of the numerical simulation of hydroplaning, two studies are of interest. The first one concerns the three-dimensional simulation of the interaction between a free surface flow and a tire [4]. In that work, the deformation of the tire shape is not considered and the computational domain remains fixed in time. The commercial FlowVision CFD code [5] is used to generate a Cartesian octreebased mesh non-conforming to the tire geometry. Due to the intersection of Cartesian cells with the tire-flow boundary, irregular polyhedral cells are generated near the domain boundaries. Few details are provided by the authors concerning the numerical treatment of the mesh. Results obtained from this investigation show a plot of lifting force acting on a tire versus angle of inclination of tread patterns placed on its surface. The second work of interest [34] proposes a model for simulating and investigating the tire hydroplaning using a three-dimensional patterned tire model provided by a home-made research code. The rainwater flow is assumed as incompressible and inviscid, and is approximated by a first-order finite-volume method and front tracking techniques. The tire shape boundary is an exchange surface between the flow solver and the tire generation code: the interaction between the tire dynamic deformation and the rainwater flow is treated by a coupling method. Cho et al. [34] simulate several patterned tires and compare the resulting contact forces. 
The numerical simulation of unsteady and incompressible isothermal multi-phase flows involving macroscopic interfaces is classically achieved thanks to the single-fluid Navier-Stokes equations $[14,36]$ and to Eulerian interface tracking methods such as the Volume Of Fluid (VOF) [7] , the Level-Set [40] or the Front Tracking approaches [41]. These methods have been extensively compared and evaluated in the last ten years and have demonstrated their qualities and drawbacks [51]. Once an interface tracking method has been chosen, the major difficulty consists in solving the motion equations for high density or viscosity ratios and large interface distortions. Near the interface, parasitic currents or unphysical flow behavior can occur when using, for example, time-splitting projection methods [21] for simulating air-water or particulate unsteady flows. In these problems, the resolution of the coupling between the incompressibility constraint and the Navier-Stokes equations is difficult to ensure in one of the phases due to the ill conditionning of the linear system or to the boundary condition treatment. Consequently, pressure-velocity coupling is the main difficulty when solving incompressible two-phase flows interacting with moving obstacles, which are involved in hydroplaning problems. The present work aims at presenting recent implicit techniques based on augmented Lagrangian methods, which improve the consistency and the accuracy of numerical simulations when unsteady multi-phase flows are dealt with. Several adaptations of the original single-phase flow augmented Lagrangian method [25] are presented for treating free surfaces and particulate flows. The major idea consists in adapting locally, in time and space, the Lagrangian or penalty parameter. The motion equations and the divergence or deformation free constraint are solved at the same time, with the same set of equations regardless of the discretization point. The formulation is based on the works of Vincent et al. $[44,48,49]$. The simulation of multiphase-flows involves ill-conditioned matrices, especially when an augmented Lagrangian approach is used. Direct solvers are well suited to treat such matrices, but they require a too large memory amount in 3D. Consequently, iterative solvers have to be used. The new Algebraic Adaptive Augmented Lagrangian (3AL) 
method is then proposed for solving multi-phase flows and improving the conditionning of the matrix when iterative solvers are implemented.

The article is structured as follows. The second section is devoted to the presentation of the models and numerical methods. Attention is paid to the description of the specific elements accounting for incompressiblity, free surface flows and fluid-structure interactions. A set of complex multi-phase flow problems is addressed in the third section, with a view of validating and demonstrating the efficiency of augmented Lagrangian techniques. Threedimensional simulations of hydroplaning flows are presented in the fourth section. In particular, the abilities of the simulation tool are evaluated on three different patterned tires which are classified according to vertical efforts. Finally, conclusions and perspectives are drawn.

\section{Numerical modeling of two-phase flows interacting with obstacles of complex shape}

\subsection{The fictitious domain approach}

The numerical simulation of a hydroplaning flow interacting with a tire could be investigated following two different numerical strategies: unstructured or structured grids. This important choice is motivated by the representation of the complex tire shape. On the one hand, the more natural solution seems to be the implementation of an unstructured body-fitted grid to simulate the area of the two-phase flow between the tire and the road. Building such a finite-volume or finite-element mesh in three-dimensions is not easy and requires automatic mesh generators as the tire moves according to time. The remeshing process at each calculation step is time consuming and can be very difficult to manage automatically in computer softwares when the shape of the tire is complex. On the other hand, it can be 
imagined to use a fixed structured grid to simulate the hydroplaning. In this case, the mesh of the fluid domain is simple. The difficulty lies in the taking into account of the complex tire shape on a non conforming grid. This type of numerical problem belongs to the class of fictitious domains [22]. The modeling strategy developed hereafter is based on this approach.

Irregular Cartesian underlaying calculation grids are considered in the rest of the article for their easy programming and the possibility to refine the road-tire interaction zone. As presented in figure 1, the fictitious domain method consists in considering each different phase (air, water and tire) as a fluid domain of specific rheological properties which is located by a phase function $C_{i}$. By definition, $C_{1}=1$ in the tire phase and 0 elsewhere whereas $C_{2}=1$ in the water phase and 0 elsewhere. The air medium is directly described by the zones where $C_{1}=0$ and $C_{2}=0$.

\subsection{The 1-fluid model}

The modeling of incompressible two-phase flows involving separated phases can be achieved by solving the incompressible Navier-Stokes equations with a phase function $C$ (in our case, $C_{2}$ is $C$. The tire shape evolutions, i.e. $C_{1}$, are obtained by coupling the CFD code to an external simulation tool, as described after). As explained by Kataoka [14], the resulting model takes implicitly into account the jumps relations at the interface $[19,36]$ and the interface evolutions are described by an advection equation on function $C$ :

$$
\begin{array}{r}
\nabla \cdot \mathbf{u}=0 \\
\rho\left(\frac{\partial \mathbf{u}}{\partial t}+(\mathbf{u} \cdot \nabla) \mathbf{u}\right)=-\nabla p+\rho \mathbf{g}+\nabla \cdot\left(\left(\mu+\mu_{t}\right)\left(\nabla \mathbf{u}+\nabla^{t} \mathbf{u}\right)\right)+\mathbf{F}_{s t} \\
\frac{\partial C}{\partial t}+\mathbf{u} \cdot \nabla C=0
\end{array}
$$


where $\mathbf{u}$ is the velocity, $p$ the pressure, $t$ the time, $\mathbf{g}$ the gravity vector, $\rho$ and $\mu$ respectively the density and the viscosity of the equivalent fluid. Concerning the turbulence modeling, a deterministic Large Eddy Simulation (LES) approach is used to take into account the underresolved sub-grid scale turbulence structures by means of a mixed scale model [32]. This model is obtained as a combination of the Smagorinsky [50] and Turbulent Kinetic Energy (TKE) [15] models. It lies on a dissipative representation, through the turbulent viscosity $\mu_{t}$, of the small scale turbulent structures. It is formulated as

$$
\mu_{T}=\rho C_{S}^{2 \alpha} C_{T K E}^{1-\alpha} \bar{\Delta}^{1+\alpha}\left(2 S_{i j} S_{i j}\right)^{\alpha / 2}\left(\frac{1}{2} \overline{\mathbf{u}^{\prime} \mathbf{u}^{\prime}}\right)^{\frac{1-\alpha}{2}}
$$

where $\alpha$ is generally chosen equal to $0.5, C_{S}$ is the Smagorinsky constant chosen in the range $0.15-0.2, C_{T K E}$ is equal to 0.2 and the fluctuation of the subgrid scales $\overline{\mathbf{u}^{\prime}}=\overline{\mathbf{u}}-\widetilde{\mathbf{u}}$ is the difference between the filtered velocity due to LES and the test filtered LES velocity. It results from the application of a specific test filter ? of compact support larger than the LES filter, applied to the LES filtered velocity $\overline{\mathbf{u}}$. The mixed scale model used in this work has been developed for single-phase flows. It is well-known that specific multi-phase LES terms should be modeled in addition to the classical inertial sub-grid terms [43]. However, we consider that in the present case (water-air) the turbulent interactions between the two phases are negligible at the sub-grid scale.

The surface tension forces are taken into account thanks to a volume force $F_{s t}=\sigma \kappa \mathbf{n}_{i} \delta_{i}$. The surface tension coefficient $\sigma$ is assumed constant. The local curvature of the interface is $\kappa$ whereas the normal to the interface is $\mathbf{n}_{i}$ and $\delta_{i}$ is a Dirac function indicating interface.

The 1-fluid model is almost identical to the classical incompressible Navier-Stokes equations, except that the local properties of the equivalent fluid ( $\rho$ and $\mu$ ) depends on $C$, the interface location requires the solving of an additional equation and a specific volume force is added at the interface to account for capillary effects. 


\subsection{Discretization and solvers}

On a general point of view, the 1-fluid Navier-Stokes equations are discretized with implicit finite-volumes on an irregular staggered Cartesian grid. A second-order centered scheme is used to approximate the spatial derivatives while a first-order Euler scheme is used for the time integration. All the terms are written at time $(n+1) \Delta t$, except the inertial term which is linearized as follows:

$$
\mathbf{u}^{n+1} \cdot \nabla \mathbf{u}^{n+1} \approx \mathbf{u}^{n} \cdot \nabla \mathbf{u}^{n+1}
$$

It has been demonstrated that this approximation allows to reach a first-order convergence in time [18], as expected. The coupling between velocity and pressure is ensured with an implicit algebraic adaptive augmented Lagrangian (3AL) method. The augmented Lagrangian methods presented in this work are independent of the chosen discretization and could be implemented for example in a finite-element framework [9]. In two-dimensions, the standard augmented Lagrangian approach [25] can be used to deal with two-phase flows as direct solvers are efficient in this case. However, as soon as three-dimensional problems are under consideration, the linear system resulting from the discretization of the augmented Lagrangian terms has to be solved with a BiCG-Stab II solver [13], preconditionned by a Modified and Incomplete LU method [12]. Indeed, in three-dimensions, the memory cost of direct solvers makes them impossible to use. The 3AL method presented bellow is a kind of general preconditionning which is particularly efficient when multi-phase or multi-material flows are undertaken, i.e. when steep density or viscosity gradients occur.

Concerning the interface tracking, a volume of fluid (VOF) approach is used with a piecewise linear interface construction (PLIC) [7]. This approach ensures the mass conservation while maintaining the interface width on one grid cell. The VOF phase function $C$ must be understood on a discrete point of view as the local volume ratio of the fluid repaired by $C$ by the total volume of the local cell. The surface tension force $F_{s t}=\sigma \kappa \mathbf{n}_{i} \delta_{i}$ is approximated 
as $F_{s t}=\sigma \nabla \cdot\left(\frac{\nabla C}{\|\nabla C\|}\right) \nabla C$, following the continuum surface force (CSF) method proposed by Brackbill et al. [20].

The numerical methods and the 1-fluid model have been widely validated by the authors concerning jet flows $[16,45]$, capillary flows $[28,33,47]$, wave breaking $[31,48,49]$, material processes [6] , plasma to water jet interaction [42] and more generally turbulent two-phase flows $[8,43]$.

\subsection{Adaptive augmented Lagrangian methods for incompressible multi-phase flows}

In the following subsections, the existing augmented Lagrangian approaches and its theoretical bases are first presented briefly in order to justify the introduction of an algebraic method for managing augmented Lagrangian techniques when multi-phase flows are dealt with $[44,49]$.

\subsubsection{Theoretical formulation}

For the sake of simplicity, let us consider the stationary incompressible Stokes equations on a domain $\Omega \in \mathbb{R}^{d}$, where $d$ is the number of space dimensions:

$$
\begin{array}{r}
-\nabla p+\mu \Delta \mathbf{u}=\mathbf{f} \text { in } \Omega \\
\nabla \cdot \mathbf{u}=0 \text { in } \Omega \\
\mathbf{u}=0 \text { on } \partial \Omega
\end{array}
$$

The functional $J(\mathbf{v})$ defined for $\mathbf{f} \in \mathrm{E}^{2}(\Omega)^{d}$ and $\mathbf{v} \in\left(H_{0}^{1}(\Omega)\right)^{d}$ is built from the weak 
formulation of the original problem:

$$
J(\mathbf{v})=\frac{\mu}{2} \mathbf{a}(\mathbf{v}, \mathbf{v})-\int_{\Omega} \mathbf{f} \cdot \mathbf{v} \mathrm{d} x=\frac{\mu}{2} \mathbf{a}(\mathbf{v}, \mathbf{v})-(\mathbf{f}, \mathbf{v})
$$

with

$$
\mathbf{a}(\mathbf{u}, \mathbf{v})=\sum_{i, j=1}^{d} \int_{\Omega} \frac{\partial u_{i}}{\partial x_{j}} \frac{\partial v_{i}}{\partial x_{j}} \mathrm{~d} x
$$

and

$$
(\mathbf{u}, \mathbf{v})=\int_{\Omega} \mathbf{u v} \mathrm{d} x
$$

This functional has to be minimized under the constraint $\mathbf{u}, \mathbf{v} \in M=\left\{\mathbf{v} \in\left(H_{0}^{1}(\Omega)\right)^{d}, \nabla \cdot \mathbf{v}=\right.$ $0\}$. The problem is now:

$$
\left\{\begin{array}{l}
J(\mathbf{u}) \leq J(\mathbf{v}), \quad \forall \mathbf{v} \in M \\
\mathbf{u} \in M
\end{array}\right.
$$

Practically, a solution in a constrained space such as $M$ cannot be easily computed. This problem of minimization under constraint is transformed into a problem of minimization without constraint thanks to a Lagrange multiplier. We define the following Lagrangian:

$$
L(\mathbf{v}, q)=J(\mathbf{v})-\int_{\Omega} q \nabla \cdot \mathbf{v} \mathrm{d} \Omega
$$

with $q \in L^{2}(\Omega)$. The minimization problem (13) consists in finding a saddle-point $(\mathbf{u}, p) \in$ $\left(H_{0}^{1}(\Omega)\right)^{d} \times L^{2}(\Omega)$ of the Lagrangian $(14):$

$$
L(\mathbf{u}, q) \leq L(\mathbf{u}, p) \leq L(\mathbf{v}, p) \quad \forall \mathbf{v} \in\left(H_{0}^{1}(\Omega)\right)^{d}, \forall q \in L^{2}(\Omega)
$$

which implies

$$
\begin{aligned}
L(\mathbf{u}, p) & =\min _{\mathbf{v} \in\left(H_{0}^{1}(\Omega)\right)^{d}} \max _{q \in L^{2}(\Omega)} L(\mathbf{v}, q) \\
& =\max _{q \in L^{2}(\Omega)} \min _{\mathbf{v} \in\left(H_{0}^{1}(\Omega)\right)^{d}} L(\mathbf{v}, q)
\end{aligned}
$$

In order to increase the convergence rate [25], the constraint is used to build a penalty term 
$\frac{1}{2} r|\nabla \cdot \mathbf{v}|^{2}, r \in \mathbb{R}[35]$. The augmented Lagrangian is denoted as:

$$
L_{r}(\mathbf{v}, q)=J(\mathbf{v})-\int_{\Omega} q \nabla \cdot \mathbf{v} \mathrm{d} x+\int_{\Omega} \frac{r}{2}|\nabla \cdot \mathbf{v}|^{2} \mathrm{~d} x .
$$

The saddle-point of $L_{r}$ is the same as for $L$ as the penalty term vanishes when the constraint $\nabla \cdot \mathbf{v}=0$ is satisfied. We admit that the solution $(\mathbf{u}, p)$ of the saddle-point problem for the weak formulation of the initial equations is the solution of the strong formulation $(7),(8)$ of the problem [25]. The saddle-point can be calculated using the Uzawa algorithm [11] :

$$
p^{0} \in \mathbb{R} \text { arbitrarily given }
$$

$p^{n}$ being known, we compute $\mathbf{u}^{n+1}$, then $p^{n+1}$ with:

$$
\begin{gathered}
L_{r}\left(\mathbf{u}^{n+1}, p^{n}\right) \leq L_{r}\left(\mathbf{v}, p^{n}\right) \quad \forall \mathbf{v} \in \mathbb{R}^{d}, \mathbf{u}^{n+1} \in \mathbb{R}^{d} \\
p^{n+1}=p^{n}+r \nabla \cdot \mathbf{u}^{n+1}
\end{gathered}
$$

Satisfying (19) is equivalent to solve

$$
-\nabla p^{n}+\mu \Delta \mathbf{u}^{n+1}+\frac{1}{2} r \nabla \cdot \mathbf{u}^{n+1}=\mathbf{f}^{n}
$$

which is the form used to build a finite-volume approximation in our approach. At this point, the value of $r$ has not been explicitly given. For Stokes equations, with constant fluid properties, Fortin and Glowinski [25] have demonstrated that the optimal value of $r$ is the average between the smaller and the larger eigenvalues of the discretization matrix. For the Navier-Stokes equations, no demonstration of optimal value are available. Without lost of generality, and for the sake of simplicity, $\frac{r}{2}$ is replaced by $r$. 


\subsubsection{Standard augmented Lagrangian (SAL)}

Following a quite similar walkthrough [25], the augmented Lagrangian can be applied to the instationary Navier-Stokes equations. The standard augmented Lagrangian (SAL) method was first introduced by Fortin and Glowinski [25]. Starting with $\mathbf{u}^{*, 0}=\mathbf{u}^{n}$ and $p^{*, 0}=p^{n}$, the solution predictor reads

while $\left\|\nabla \cdot \mathbf{u}^{*, \mathbf{m}}\right\|>\epsilon$, solve

$$
\begin{gathered}
\left(\mathbf{u}^{*, 0}, p^{*, 0}\right)=\left(\mathbf{u}^{n}, p^{n}\right) \\
\rho\left(\frac{\mathbf{u}^{*, m}-\mathbf{u}^{*, 0}}{\Delta t}+\mathbf{u}^{*, m-1} \cdot \nabla \mathbf{u}^{*, m}\right)-r \nabla\left(\nabla \cdot \mathbf{u}^{*, m}\right) \\
=-\nabla p^{*, m-1}+\rho \mathbf{g}+\nabla \cdot\left[\mu\left(\nabla \mathbf{u}^{*, m}+\nabla^{T} \mathbf{u}^{*, m}\right)\right]+\sigma k \mathbf{n}_{i} \delta_{i} \\
p^{*, m}=p^{*, m-1}-r \nabla \cdot \mathbf{u}^{*, m}
\end{gathered}
$$

where $r$ is the augmented Lagrangian parameter used to impose the incompressibility constraint, $m$ is an iterative convergence index and $\epsilon$ a numerical threshold controlling the constraint. Usually, a constant value of $r$ is used. From numerical experiments, optimal values are found to be of the order of $\rho_{i}$ and $\mu_{i}$ to accurately solve the motion equations in the related zone [49]. The momentum, as well as the continuity equations are accurately described by the predictor solution $\left(\mathbf{u}^{*}, p^{*}\right)$ coming from $(22)$ in the medium, where the value of $r$ is adapted. However, high values of $r$ in the other zones act as penalty terms inducing the numerical solution to satisfy the divergence-free property only. Indeed, if we consider for example $\rho_{1} / \rho_{0}=1000$ (characteristic of water and air problems) and a constant $r=\rho_{1}$ to impose the divergence-free property in the denser fluid, the asymptotic equation system 
solved in the predictor step is:

$$
\begin{aligned}
& \rho\left(\frac{\mathbf{u}^{*}-\mathbf{u}^{n}}{\Delta t}+\left(\mathbf{u}^{n} \cdot \nabla\right) \mathbf{u}^{*}\right)-r \nabla\left(\nabla \cdot \mathbf{u}^{*}\right) \\
& =\rho \mathbf{g}-\nabla p^{n}+\nabla \cdot\left[\mu\left(\nabla \mathbf{u}^{*}+\nabla^{T} \mathbf{u}^{*}\right)\right]+\sigma k \mathbf{n}_{i} \delta_{i} \quad \text { in } \Omega_{1} \\
& \frac{\mathbf{u}^{*}-\mathbf{u}^{n}}{\Delta t}-r \nabla\left(\nabla \cdot \mathbf{u}^{*}\right)=0 \quad \text { in } \Omega_{0}
\end{aligned}
$$

Our idea is to locally estimate the augmented Lagrangian parameter in order to obtain satisfactory equivalent models and solutions in all the media.

\subsubsection{Adaptive augmented Lagrangian (2AL)}

Instead of choosing an empirical constant value of $r$ fixed at the beginning of the simulations, we propose at each time step to locally estimate the augmented Lagrangian parameter $r$. Then, $r(t, M)$ becomes a function of time $t$ and space position $M$. It must be two to three orders of magnitude higher than the most important term in the conservation equations.

Let $L_{0}, t_{0}, u_{0}$ and $p_{0}$ be reference space length, time, velocity and pressure respectively. If we consider one iterative step of the augmented Lagrangian procedure (22), the non-dimensional form of the momentum equations can be rewritten as

$$
\begin{aligned}
& \rho \frac{u_{0}}{t_{0}} \frac{\mathbf{u}^{*, m}-\mathbf{u}^{n}}{\Delta t}+\rho \frac{u_{0}^{2}}{L_{0}}\left(\mathbf{u}^{*, m-1} \cdot \nabla\right) \mathbf{u}^{*, m}-\frac{u_{0}}{L_{0}^{2}} \nabla\left(r \nabla \cdot \mathbf{u}^{*, m}\right) \\
= & \rho \mathbf{g}-\frac{p_{0}}{L_{0}} \nabla p^{*, m-1}+\frac{u_{0}}{L_{0}^{2}} \nabla \cdot\left[\mu\left(\nabla \mathbf{u}^{*, m}+\nabla^{T} \mathbf{u}^{*, m}\right)\right]+\frac{\sigma}{L_{0}^{2}} k \mathbf{n}_{i} \delta_{i}
\end{aligned}
$$

Multiplying the right and left parts of equation (24) by $L_{0}^{2} / u_{0}$, we can compare the augmented Lagrangian parameter $r$ to all the contributions of the flow (inertia, gravity, pressure and viscosity). We obtain

$$
\begin{gathered}
\\
\rho \frac{L_{0}^{2}}{t_{0}} \frac{\mathbf{u}^{*, m}-\mathbf{u}^{n}}{\Delta t}+\rho u_{0} L_{0}\left(\mathbf{u}^{*, m-1} \cdot \nabla\right) \mathbf{u}^{*, m}-\nabla\left(r \nabla \cdot \mathbf{u}^{*, m}\right) \\
=\rho \frac{L_{0}^{2}}{u_{0}} \mathbf{g}-\frac{p_{0} L_{0}}{u_{0}} \nabla p^{*, m-1}+\nabla \cdot\left[\mu\left(\nabla \mathbf{u}^{*, m}+\nabla^{T} \mathbf{u}^{*, m}\right)\right]+\frac{\sigma}{u_{0}} k \mathbf{n}_{i} \delta_{i}
\end{gathered}
$$


It can be noticed that $r$ is comparable to a viscosity coefficient. It is then defined as

$$
r(t, M)=\mathcal{K} \max \left(\rho(t, M) \frac{L_{0}^{2}}{t_{0}}, \rho(t, M) u_{0} L_{0}, \rho(t, M) \frac{L_{0}^{2}}{u_{0}} g, \frac{p_{0} L_{0}}{u_{0}}, \mu(t, M), \frac{\sigma}{u_{0}}\right)
$$

If, for example, $\frac{\rho_{1}}{\rho_{0}}=1000$ and $\mu_{0}<\mu_{1}<<\rho_{0}<<\rho_{1}$, the semi-discrete form of the momentum equations resulting from the new values of $r(t, M)$ given by (26) then becomes

$$
\begin{aligned}
& \rho_{1}\left(\frac{\mathbf{u}^{*, m}-\mathbf{u}^{n}}{\Delta t}+\left(\mathbf{u}^{*, m-1} \cdot \nabla\right) \mathbf{u}^{*, m}\right)-\nabla\left(r \nabla \cdot \mathbf{u}^{*, m}\right) \\
& =\rho_{1} \mathbf{g}-\nabla p^{*, m-1}+\nabla \cdot\left[\mu_{1}\left(\nabla \mathbf{u}^{*, m}+\nabla^{T} \mathbf{u}^{*, m}\right)\right]+\sigma k \mathbf{n}_{i} \delta_{i} \\
& \quad \text { in } \Omega_{1} \text { with } r=\mathcal{K}_{1} \rho_{1} L_{0}^{2} u_{0} \\
& \rho_{0}\left(\frac{\mathbf{u}^{*, m}-\mathbf{u}^{n}}{\Delta t}+\left(\mathbf{u}^{*, m-1} \cdot \nabla\right) \mathbf{u}^{*, m}\right)-\nabla\left(r \nabla \cdot \mathbf{u}^{*, m}\right) \\
& =\rho_{0} \mathbf{g}-\nabla p^{*, m-1}+\nabla \cdot\left[\mu_{0}\left(\nabla \mathbf{u}^{*, m}+\nabla^{T} \mathbf{u}^{*, m}\right)\right]+\sigma k \mathbf{n}_{i} \delta_{i} \\
& \text { in } \Omega_{0} \text { with } r=\mathcal{K}_{0} \rho_{0} L_{0}^{2} u_{0}
\end{aligned}
$$

where $\mathcal{K}_{0}$ and $\mathcal{K}_{1}$ are in between 10 and 1000. In this way, thanks to expression (25), the adaptive Lagrangian parameter is assigned a value that exceeds from 10 to 1000 times the order of magnitude of the most important term between inertia, viscosity, pressure or gravity in both $\Omega_{1}$ and $\Omega_{0}$ domains. Compared to the SAL approach (23), the $2 \mathrm{AL}$ is consistent with the Navier-Stokes equations in each phase [49]. The new method (26) can be easily extended to other forces such as Coriolis effects or specific source terms. Comparisons between standard and adaptive augmented Lagrangian (2AL) methods are presented in the next section. In particular, the influence of the penalty parameter on the convergence speed of the BiCG-Stab solver and time and space variations of $r(M)$ are discussed.

As a summary, the complete time-marching procedure of the predictor-corrector algorithm including the $2 \mathrm{AL}$ method is the following: 
Step 1: initial values $\mathbf{u}^{0}$ and $p^{0}$ and boundary conditions on $\Gamma$ are defined,

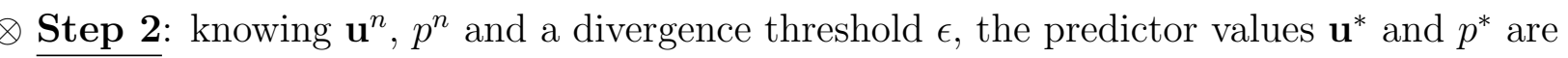
estimated with the Uzawa algorithm (22) associated to the local estimate of $r(t, M)$ defined in expression (26), so that $\mathbf{u}^{*}=\mathbf{u}^{*, m}$ and $p^{*}=p^{*, m}$ when $m$ verifies $\left\|\nabla \cdot \mathbf{u}^{*, \mathbf{m}}\right\|<\epsilon$,

$\otimes \underline{\text { Step 3: }}$ the solution $\left(\mathbf{u}^{*}, p^{*}\right)$ is projected on a divergence-free subspace thanks, for example, to projection approaches $[18,21]$ to get the correction solution $\left(\mathbf{u}^{\prime}, p^{\prime}\right)$. Then, the numerical solution at time $(n+1) \Delta t$ is $\left(\mathbf{u}^{n+1}, p^{n+1}\right)=\left(\mathbf{u}^{*}+\mathbf{u}^{\prime}, p^{*}+p^{\prime}\right)$,

$\otimes$ Step 4: $n$ is iterated in steps 3 and 4 until the physical time is reached.

\subsubsection{Algebraic adaptive augmented Lagrangian (3AL)}

It has been demonstrated that estimating a local and adapted augmented Lagrangian parameter is crucial for simulating multi-phase flows [49]. The main remaining drawback of the 2AL method is linked to the a priori definition of dimensionless parameters for defining $r(t, M)$. The augmented Lagrangian approach is based on the concept of a penalty method. As a consequence, the augmented Lagrangian parameter acts as an algebraic parameter which increases the magnitude of specific coefficients in the linear system in order to verify a specific constraint, while solving at same time the conservation equations. In this section, an estimate of $r(t, M)$ is proposed which is based on a scanning of the matrix coefficients. The main interests of the algebraic adaptive augmented Lagrangian method (3AL) are the following: it does not require any a priori physical information, it applies to any kind of geometry and grid and it takes into account the residual of the linear solver and the fulfilment of incompressible and solid constraints. 
At each time step and in two-dimensions (the three-dimensional algorithm is straightforward), the 3AL method determines $r(t, M)$ as follows:

$\otimes \underline{\text { Step 1: }}$ two matrices $A$ and $A^{*}$ are built corresponding respectively to the discretization of the momentum equations (27) with $r(t, M)=0$ and $r(t, M)=1$. In order to optimize computer memory, a compressed storage raw (CSR) structure is chosen to store only the non-null coefficients of each matrix,

$\otimes \underline{\text { Step 2: }}$ on the fixed staggered Cartesian grid, $r(t)_{i, j}$ is evaluated according to the discretization coefficients of the surrounding velocity $u_{x, i-\frac{1}{2}, j}, u_{x, i+\frac{1}{2}, j}, u_{y, i, j-\frac{1}{2}}$ and $u_{y, i, j+\frac{1}{2}}$ components, as presented in figure 2. The discretization of each velocity component, $u_{x, i-\frac{1}{2}, j}$ for example, requires the use of 9 neighboring velocity nodes, i.e. $u_{x, i-\frac{1}{2}, j}, u_{x, i+\frac{1}{2}, j}, u_{x, i-\frac{3}{2}, j}$, $u_{x, i-\frac{1}{2}, j+1}$ and $u_{x, i-\frac{1}{2}, j-1}$ for the discretization of the inertial and viscous terms and $u_{y, i-1, j-\frac{1}{2}}$, $u_{y, i-1, j+\frac{1}{2}}, u_{y, i, j-\frac{1}{2}}$ and $u_{y, i, j+\frac{1}{2}}$ for the viscous and augmented Lagrangian terms. In this way, we estimate the maximum values of the discretization coefficients $A_{I}(u), 1 \leqslant I \leqslant 9$, associated to velocities $u_{x, i-\frac{1}{2}, j}, u_{x, i+\frac{1}{2}, j}, u_{y, i, j-\frac{1}{2}}$ and $u_{y, i, j+\frac{1}{2}}$. We define:

$$
\left\{\begin{array}{c}
C_{i-\frac{1}{2}, j}=\max _{I=1 \ldots 9}\left|A_{I}\left(u_{x, i-\frac{1}{2}, j}\right)\right| \\
C_{i+\frac{1}{2}, j}=\max _{I=1 \ldots 9}\left|A_{I}\left(u_{x, i+\frac{1}{2}, j}\right)\right| \\
C_{i, j-\frac{1}{2}}=\max _{I=1 \ldots 9}\left|A_{I}\left(u_{y, i, j-\frac{1}{2}}\right)\right| \\
C_{i, j+\frac{1}{2}}=\max _{I=1 \ldots 9}\left|A_{I}\left(u_{y, i, j+\frac{1}{2}}\right)\right|
\end{array}\right.
$$

$\underline{\text { Step 3: }}$ in the same way, the coefficient $C_{i-\frac{1}{2}, j}^{*}, C_{i+\frac{1}{2}, j}^{*}, C_{i, j-\frac{1}{2}}^{*}$ and $C_{i, j+\frac{1}{2}}^{*}$, corresponding to $A^{*}$, are estimated,

Step 4: the minimum and maximum discrete coefficient $\operatorname{Rmin}_{i, j}$ and $\operatorname{Rmax}_{i, j}$ at the 
scalar position of $r(t)_{i, j}$ are then defined as:

$$
\left\{\begin{array}{l}
\operatorname{Rmin}_{i, j}=\min \left(\left|\frac{C_{i-\frac{1}{2}, j}}{C_{i-\frac{1}{2}, j}^{*}}\right|,\left|\frac{C_{i+\frac{1}{2}, j}}{C_{i+\frac{1}{2}, j}^{*}}\right|,\left|\frac{C_{i, j-\frac{1}{2}}}{C_{i, j-\frac{1}{2}}^{*}}\right|,\left|\frac{C_{i, j+\frac{1}{2}}}{C_{i, j+\frac{1}{2}}^{*}}\right|\right) \\
\operatorname{Rmax}_{i, j}=\max \left(\left|\frac{C_{i-\frac{1}{2}, j}}{C_{i-\frac{1}{2}, j}^{*}}\right|,\left|\frac{C_{i+\frac{1}{2}, j}}{C_{i+\frac{1}{2}, j}^{*}}\right|,\left|\frac{C_{i, j-\frac{1}{2}}}{C_{i, j-\frac{1}{2}}^{*}}\right|,\left|\frac{C_{i, j+\frac{1}{2}}}{C_{i, j+\frac{1}{2}}^{*}}\right|\right)
\end{array}\right.
$$

$\otimes \underline{\text { Step 5: }}$ as can be seen in figure 2, the parameters $r(t)_{i, j}$ and $r^{*}(t)_{i, j}$ are located at pressure nodes while the $C$ and $C^{*}$ are located at velocity nodes (in the staggered grid approach). In the calculation of Rmin and $R \max$, strong variations of $C$ and $C^{*}$ can occur in the vicinity of air-gaz or fluid-solid interfaces. Without modification, the penalty of the incompressible or solid constraint is not ensured due to grid irregularity or strong local variation of physical or penalty parameters. In this way, a threshold is used for $r^{*}(t)_{i, j}$ : if $\frac{\operatorname{Rmax}_{i, j}}{\operatorname{Rmin}_{i, j}} \leqslant 10^{10}$ and $\frac{\max \left(\Delta_{x, i, j}, \Delta_{y, i, j}\right)}{\min \left(\Delta_{x, i, j}, \Delta_{y, i, j}\right)} \leqslant 1000, r^{*}(t)_{i, j}=\operatorname{Rmin}_{i, j}$. Else, $r^{*}(t)_{i, j}=\operatorname{Rmax}_{i, j}$,

Q Step 6: once $r^{*}(t)_{i, j}$ has been estimated for all $i$ and $j, 1 \leqslant i \leqslant n_{x}$ and $1 \leqslant j \leqslant n_{y}$, the local values of the algebraic penalty parameters are normalized as $r(t)_{i, j}=\frac{\mathcal{K} r^{*}(t)_{i, j}}{r_{m i n}^{*}+10^{-40}}$, where $r_{\text {min }}^{*}=\min _{i=1 . . n_{x}, j=1 . . n_{y}}\left(r^{*}(t)_{i, j}\right)$. As for the SAL and the $2 \mathrm{AL}, \mathcal{K}$ is a numerical parameter used to tune the minimization of the divergence according to the residual of the solver. The higher $\mathcal{K}$ is, the lower is the divergence and higher the residual is. In the $3 \mathrm{AL}$, the order of magnitude of $r(t)_{i, j}$ is naturally balanced so the constant $\mathcal{K}$ is equal to 1 except at the first calculation step where $\mathcal{K}=100$ if $\frac{\operatorname{Rmax}_{i, j}}{\operatorname{Rmin}_{i, j}} \leqslant 10^{10}$ or if during the five first calculation steps, the norm of the residual of the linear solver divided by the norm of the divergence, obtained at the previous calculation step, is greater than $10^{6}$. This last particular case can be obtained when a flow simulation is initialized without any imposed velocity field.

Steps 1 to 6 are repeated at each calculation step corresponding to a physical time incremented of $\Delta t$ seconds. 


\subsection{Management of moving obstacles by penalty methods}

While a phase function $C_{i}$ is used to locate the different fluid media, a binary Heaviside function $\chi_{1}$ is used to locate the cells for which their center is inside the solid, and consequently $\chi_{1}=1$ in the solid, i.e. the tire in the present work, and 0 elsewhere.

The principle of the penalty method is to use a unique set of equation in the whole computational domain and to add specific penalty terms to the initial physical model. The magnitude of the penalty terms varies according to $\chi_{1}$ in order to impose a particular behavior in each media. The Brinkman penalty term [22] accounting for Darcy flows in solid porous media is one of the simplest penalty method for the Navier-Stokes equations. It consists in adding the term $\frac{\mu}{K} \mathbf{u}$ in the momentum conservation equation, with $K$ the local permeability. The equation (3) becomes:

$$
\rho\left(\frac{\partial \mathbf{u}}{\partial t}+(\mathbf{u} \cdot \nabla) \mathbf{u}\right)+\frac{\mu}{K} \mathbf{u}=-\nabla p+\rho \mathbf{g}+\nabla \cdot\left(\mu\left(\nabla \mathbf{u}+\nabla^{t} \mathbf{u}\right)\right)+\mathbf{F}_{s t}
$$

In the fluid media $\left(\chi_{1}=0\right), K \rightarrow+\infty$ and the penalty term has no effect. In the solid media $\left(\chi_{1}=1\right), K \rightarrow 0$ and the equation $(28)$ reduces to $\mathbf{u}=0$. Using a Brinkman penalty term in this way is a staightforward method for modeling fixed obstacles. For moving obstacles such as a tire, a volume penalty method [2] is more suitable. The Brinkman penalty term is replaced in the equation (28) by $\frac{\chi_{1}}{\varepsilon}\left(\mathbf{u}-\mathbf{u}_{D}\right)$ with $\mathbf{u}_{D}$ the local prescribed velocity of the tire, $\varepsilon$ the local penalty coefficient and $\chi_{1}$ the Heaviside function related to $\Omega_{2}$. As $\varepsilon \ll 1$, the penalized momentum equations tends to $\mathbf{u}=\mathbf{u}_{D}$ where $\chi_{1}=1$ (i.e. in the solid domain) and a non-zero velocity can be imposed.

These methods are first order in space only as each local velocity is imposed in an entire grid cell. Hence, the original shape of a complex object is considered as being a set of boxes matching the control volumes of the Eulerian grid. The first order penalty method is used in this work for its easy programming. A more accurate description of the interface can be obtained with the sub-mesh penalty method $[2,3]$. This more complex method takes into 
account the location of the interface and reaches a second order in space.

These penalty methods are used to treat one-way coupling fluid-structure interaction in which the velocity field in the solid is known a priori. They are used to simulate hydroplaning.

Concerning the initialization of the object function $\chi_{1}$, a ray-casting method is used [3]. For each point of the Eulerian grid, a ray is casted to the infinity. If the number of intersections between the ray and the Lagrangian surface grid $\Sigma_{h}$ describing the obstacle is even, the point is outside of the object, neither inside. The whole methodology, especially for curvilinear structured grids, is detailed in a recent work [1].

\subsection{Calculation of the force exerted on obstacles}

The fluid force applied on an obstacle such as the tire is

$$
F=\int_{\Sigma} \boldsymbol{\sigma} \cdot \mathbf{n} \mathrm{d} S=\int_{\Sigma}(2 \mu D-p I d) \cdot \mathbf{n} \mathrm{d} S
$$

where $I d$ is the identity matrix, $\mathrm{d} S$ the surface unit, $\boldsymbol{\sigma}$ is the stress tensor, $D$ the deformation tensor and $\mathbf{n}$ the local normal to the fluid-solid interface. The resulting force is decomposed in two parts:

$$
\begin{gathered}
F^{p}=-\int_{\Sigma}(p I d) \cdot \mathbf{n} d S \\
F^{v}=\int_{\Sigma}(2 \mu D) \cdot \mathbf{n} d S
\end{gathered}
$$

with $F^{p}$ the pressure force and $F^{v}$ the viscous force.

The numerical computation of the wall forces are often used in the literature of the fictitious domain methods where the accuracy of the drag force of a particle is a common test case. Nonetheless, the details of such a calculation are rarely explained. For the Brinkman penalty approach, the volume integral method proposed by Caltagirone [17] is simple and 
well suited. When volume penalty fictitious domain techniques of first or higher orders are used, a different calculation is desirable.

The theoretical force calculation requires to know the pressure and the deformation tensor of the velocity on the fluid-obstacle interface. The tire surface is discretized as a Lagrangian surface composed of triangular elements. The fluid field is only defined outside of the object (exceptions will be discussed later) and the interface does not generally match the location of the Eulerian nodes. As a consequence, a quantity has to be extrapolated from the fluid domain to the interface and the first step is to define points in the fluid to build the interpolations. The number of surface element $\sigma_{i}$ of $\Sigma_{h}$ (the discretization of $\Sigma$ ) is denoted as $N_{e}$. In three-dimensions, the elements are triangles defined by three points of coordinates $\mathbf{x}_{i k}^{e}, k=1 . .3$. For each element $\sigma_{i}, 1 \leqslant i \leqslant N_{e}$, we define $\mathbf{x}_{i}=\frac{\sum_{1 \leqslant k \leqslant 3} \mathbf{x}_{i k}^{e}}{3}$ the barycenter of the element and $\tilde{\mathbf{n}}_{i}$ its outward local normal at $\mathbf{x}_{i}$. Three fluid extrapolation points are then defined at the locations

$$
\mathbf{x}_{i l}=\mathbf{x}_{i}+l(\Delta x \Delta y \Delta z)^{1 / 3} \tilde{\mathbf{n}}_{i}, l=1, . ., 3
$$

The discrete values of a given field $\Phi$ (pressure, derivative...) are then interpolated on the Lagrangian points $\mathbf{x}_{i l}$. For each $\mathbf{x}_{i l}$, the Eulerian grid points used to interpolate $\Phi$ in $\mathbf{x}_{i l}$ are denoted $\mathbf{x}_{i l}^{j}, j=1, \ldots, S_{\max }$ with $S_{\max }$ the number of Eulerian grid points of the stencil of the considered interpolation function. Using two or three Lagrangian points and more related Eulerian grid points to interpolate $\Phi$ on $\sigma_{i}$ produces an interpolation with a large stencil. Furthermore, each extrapolated value of a component of $D$ is itself a centered derivative which enlarges again the stencil. Hence, two constraints opposed themselves:

- the calculation and the interpolation at the points $x_{i l}$ of the stress tensor requires a large stencil, so the tensor has not to be taken too close to the considered element $\sigma_{i}$

- a point inside the solid could be accidently used to compute the extrapolated values of $D$ if the Lagrangian points $x_{i k}$ is taken too far from $\sigma_{i}$ 
To a smaller degree, these constraints remain valid for the computation of the pressure at the interface. The occurrence of such problems varies according to the complexity of the discrete Lagrangian interface $\Sigma_{h}$. A convex shape does not generally induces such effects and the study of the wall forces on a sphere is easily performed. For shapes with concavity such as a tire with complex patterns, the troubles increases with the curvature of the shape.

However, if the ratio of the magnitudes of the pressure forces to the viscous forces is such that the viscous forces are negligible, the wall forces calculation is easier to perform. The pressure field is directly available on the grid, and one can simply take its value at the closest fluid node. If a calculation of higher order of the pressure is required, two methods can be used to prevent the troubles induced by the curvature of the interface.

The first method, which is utilized in the present paper, is to use adaptive interpolations such as kernel functions [37] which are built with any number of points belonging to a restricted spherical area of chosen radius $R$ surrounding $x_{i k}$. For example, $R=$ $\max (\Delta x, \Delta y, \Delta z)$. To be efficient, only the fluid points such as $\chi_{1}<0.5$ are considered in this approach.

The second method is to extend the field $\Phi$ from the fluid media to the solid media. The resulting field denoted as $\tilde{\Phi}$ allows the use of standard Lagrange interpolation schemes which select Eulerian values in the obstacle region as $\tilde{\Phi}$ is continuous across $\Sigma_{h}$. The following penalty Helmoltz equation is solved to obtain $\tilde{\Phi}$ :

$$
\nabla^{2} \tilde{\Phi}+\frac{1}{\varepsilon_{p}}\left(\tilde{\Phi}-\Phi^{n+1}\right)=0
$$

where $\Phi^{n+1}$ is the value obtained by solving the conservation equations and $\varepsilon_{p}$ is a penalty parameter tending to infinity if $\chi_{1} \geq 0.5$ and to 0 if $\chi_{1}<0.5$. As a resulting property, the physical values of $\Phi^{n+1}$ are maintained in the fluid and $\tilde{\Phi}$ is continuous across $\Sigma_{h}$. Then, $\tilde{\Phi}$ can be interpolated from the Eulerian grid to $\Sigma_{h}$. In this case, a $Q_{1}$ interpolation can be used. In $2 \mathrm{D}$ it reads $Q_{1}(x, y)=a x y+b x+c y+d$, while in $3 \mathrm{D}$, 
$Q_{1}(x, y, z)=a x y z+b x y+c y z+d z x+e x+f y+g z+h$. As a Neumann condition is not imposed on $\Sigma$ for the problem (33), the first derivative of $\tilde{\Phi}$ is not continuous. Hence, if $\tilde{\Phi}$ is the velocity, one cannot use $\tilde{\Phi}$ to calculate $D$. As can be seen, the calculation of a high order interpolation of $D$ on the interface is a complex problem which requires the use of a high order local penalty method [2] to impose in (33) that $\nabla \tilde{\Phi} \cdot \tilde{\mathbf{n}}_{i}=0$ on $\Sigma_{h}$ in the obstacle. This point is not presented here as fortunately, the viscous forces are negligible in the case of the hydroplaning of a tire treated in the present paper.

Once the Eulerian extended pressure $\tilde{p}$ is estimated by (33), the pressure force $F^{p}$ is implemented by a discretization of (30) as follows:

$$
F^{p}=-\sum_{1 \leqslant i \leqslant N_{e}} \tilde{p}_{i} \Delta S_{i} \tilde{\mathbf{n}}_{i}
$$

where $\tilde{p}_{i}$ are extrapolated from the fluid domain to the surface with

$$
\tilde{p}_{i}=3 \tilde{p}_{i 1}-3 \tilde{p}_{i 2}+\tilde{p}_{i 3}
$$

and

$$
\tilde{p}_{i l}=\frac{\sum_{1 \leqslant j \leqslant S_{\max }} \tilde{p}_{i l}^{j} d_{i l}^{j}}{\sum_{1 \leqslant j \leqslant S_{\max }} d_{i l}^{j}}, l=1, . ., 3
$$

The notation ${ }_{\cdot i l}^{j}$ corresponds to a value taken at the Eulerian nodes $\mathbf{x}_{i l}^{j}$ surrounding the Lagrangian position $\mathbf{x}_{i l}$. The surface of element $\sigma_{i}$ is

$$
\Delta S_{i}=\frac{1}{2} \sqrt{\tilde{\mathbf{n}}_{x, i}^{2}+\tilde{\mathbf{n}}_{y, i}^{2}+\tilde{\mathbf{n}}_{z, i}^{2}}
$$

where $\tilde{\mathbf{n}}_{x, i}, \tilde{\mathbf{n}}_{y, i}$ and $\tilde{\mathbf{n}}_{z, i}$ are the components of $\tilde{\mathbf{n}}_{i}$. The notation $d_{i l}^{j}$ denotes the distance between the Lagrangian barycenter $\mathbf{x}_{i}$ and the Eulerian position $\mathbf{x}_{i l}^{j}$ whereas the outward local normal $\tilde{\mathbf{n}}_{i}$ is estimated at the barycenter $\mathbf{x}_{i}$ of each $\sigma_{i}$ by taking the vectorial product 
of two tangent vectors $\tilde{\mathbf{t}}_{i, 1}$ and $\tilde{\mathbf{t}}_{i, 2}$ to $\sigma_{i}$ :

$$
\tilde{\mathbf{n}}_{i}=\tilde{\mathbf{t}}_{i, 1} \wedge \tilde{\mathbf{t}}_{i, 2}
$$

For example, the tangent vectors can be defined as

$$
\begin{aligned}
& \tilde{\mathbf{t}}_{i, 1}=\left(\begin{array}{c}
\mathbf{x}_{i 2, x}^{e}-\mathbf{x}_{i 1, x}^{e} \\
\mathbf{x}_{i 2, y}^{e}-\mathbf{x}_{i 1, y}^{e} \\
\mathbf{x}_{i 2, z}^{e}-\mathbf{x}_{i 1, z}^{e}
\end{array}\right) \\
& \tilde{\mathbf{t}}_{i, 2}=\left(\begin{array}{c}
\mathbf{x}_{i 3, x}^{e}-\mathbf{x}_{i 1, x}^{e} \\
\mathbf{x}_{i 3, y}^{e}-\mathbf{x}_{i 1, y}^{e} \\
\mathbf{x}_{i 3, z}^{e}-\mathbf{x}_{i 1, z}^{e}
\end{array}\right)
\end{aligned}
$$

where $\mathbf{x}_{i k, x}^{e}, \mathbf{x}_{i k, y}^{e}$ and $\mathbf{x}_{i k, z}^{e}, k=1 . .3$, denotes the Cartesian coordinates of the points forming the element $\sigma_{i}$.

\section{Numerical testing and validation of augmented La- grangian methods}

The augmented Lagrangian methods have been widely validated and compared to experimental and theoretical reference results in previous publications [10,48, 49]. For example, the 2D laplace law, the sedimentation of a particle in Stokes regimes, the flow across a fixed cubic face centered (CFC) array of particles and the two-dimensional wave breaking in deep water have been investigated. The $2 \mathrm{AL}$ method has proven its efficiency and its consistency with physical and numerical reference solutions. In this section, the $3 \mathrm{AL}$ approach is investigated and validated on reference single- and two-phase flows. No additional projection step is used as the purpose here is to evaluate the AL approach.

If not specified, the 2D cases are solved with a direct PARDISO solver [29]. 


\subsection{Square thermally driven cavity at high Rayleigh number}

In order to avoid the error due to the interface tracking, a single phase flow is first considered. This test case is the square thermally driven cavity at high Rayleigh number. The objective is to evaluate the intrinsic space convergence order of the $3 \mathrm{AL}$. The same physical parameters as in [46] are used. The Rayleigh number $R a=\left(\mathbf{g} \beta \Delta T H^{3}\right) /(\nu \lambda)$ (with $\Delta T$ the difference of temperature between the two vertical walls, $H$ the wall length, $\nu$ the dynamic viscosity and $\lambda$ the thermal conductivity) is characteristic of the naturel convection problems. Three Rayleigh numbers $10^{5}, 10^{6}$ and $10^{7}$ are studied, corresponding to laminar conditions. Very accurate solutions of the problem for $10^{5} \leq R a \leq 10^{8}$ were obtained by Le Quéré [30] using spectral methods. Nusselt numbers are given for the Rayleigh numbers presently considered and can be taken as reference values. However, the Nusselt numbers given in [30] cannot be used for a precise convergence study as Le Quéré does not give enough decimals. A Richardson extrapolation [24] is then used to determine the numerical convergence solutions. The table 1 compares the Nusselt numbers given by [30] and the numbers found here with the Richardson extrapolation. The convergence orders given in table 1 are obtained from the Richardson analysis. As can be seen, a very good agreement is obtained. These extrapolated values are taken as reference values in figure 3 which shows the spatial convergence of the error on the Nusselt number. As can be seen, the convergence is very regular and the space convergence order is two. To finish with, the figure 4 shows the local values of $r(t, M)$ for the case $R a=10^{5}$ on a $512 \times 512$ mesh. In this case, as for every Rayleigh number, the iso-lines of $r(t, M)$ are following the temperature map. This parameter which is determined with an algebraic method is sensitive to the density variation in this case.

\section{$3.22 \mathrm{D}$ collapse of a water column}

The ability of the 3AL method to simulate free surface flows is investigated on the collapse of a $2 \mathrm{D}$ water column in a tank. The interest of this two-phase flow problem is to provide 
experimental measurements of free surface position at several characteristic times [39]. The width and height of the calculation domain are $0.584 \mathrm{~m}$ and $1.168 \mathrm{~m}$ respectively. As presented in figure 5, the closed cavity is initially full of air and the water column is placed in the bottom left corner of the domain. Its width and height are $0.146 \mathrm{~m}$ and $0.292 \mathrm{~m}$.

A convergence study is carried out on three calculation grids $200 \times 400,400 \times 800$ and $800 \times 1600$. The time steps used for each grid simulation are $5 \times 10^{-4} s, 2.5 \times 10^{-4} s$ and $1.25 \times 10^{-4} \mathrm{~s}$. Ten calculation steps are used in the iterative BiCG-Stab II solver. The results are presented in figure 6. The experimental visualizations of Koshizuka and Oka [39] have been digitalized in order to extract the position of the free surface at several points. It is observed that all the simulations describe correctly the dynamics of the water which runs until $t=0.04 \mathrm{~s}$. From $t=0.6 \mathrm{~s}$ to $t=0.8 \mathrm{~s}$, the left part of the flow is accurately recovered by all grid calculations. However, near the right wall, significant discrepancies are observed between numerical simulations and experiments. The finest grid provides the best description of the flow near the right wall, where the water film formed on the wall falls under the action of gravity, hits the water on the bottom boundary and splashes. The flow is strongly non linear, with important interface deformation, coalescence and rupture. On a macroscopic point of view, the three grids provide solutions which are in good agreement with the experimental measurements, demonstrating the independency of most of the interfacial flow structures with respect to the grid.

Concerning the magnitude and the evolution of the 3AL parameter $r(t, M)$, they are illustrated in figure 7 for simulations leaded on a $400 \times 800$ grid. At each calculation step, the local magnitude of $r(t, M)$ evolves and adapts in order to ensure the divergence-free constraint in a balanced way. In the air medium, $r(t, M)$ is almost equal to 1 , while $r(t, M)$ lies between 100 and 400 in water.

Eventually, we evaluate the SAL method for the collapse problem of a water column. On 
figure 8 , a $400 \times 800$ grid is considered and the simulation results are discussed after the jet formed on the right wall has splashed down. Three simulations have been performed, with constant values of $r(t, M)$, equal to 1000 and 100, and 10 and 50 iterations of the iterative BiCG-Stab II solver. It is observed that with the same numerical cost of the iterative solver as with the 3AL method (10 iterations), the numerical solution obtain with $r(t, M)=1000$ is the worst result of the three (black line) while by changing $r(t, M)$ to 100 , the simulation get closest to the experimental measurements and to the 3AL simulation. Increasing the number of BiCG iteration, while keeping $r(t, M)=1000$, slightly improves the results but the numerical cost of the simulation is multiplied by 5. A comparison on the numerical performances according to the method used is provided in table 2 on a $400 \times 800$ grid. It is observed that the 3AL approach involves a negligible overcost compared to SAL, while the residual and divergence obtained with the same solver are comparable as soon as the number of internal solving steps of the iterative method reaches 50. The major difference lies in the physical meaning of the obtained solutions, which are always better with $3 \mathrm{AL}$ than with SAL, even for only 5 or 10 BiCG-Stab II iterations.

To conclude, the collapse problem of a water column allowed us to demonstrate the interest of the 3AL method, compared to SAL approaches, as well as 2AL techniques: the 3AL method is always well balanced concerning incompressible penalty terms and requires less number of solver iterations to get a suitable solution than SAL and 2AL approaches. In addition, contrary to $2 \mathrm{AL}$ approach, the $3 \mathrm{AL}$ method is not based on any a priori physical information and does not involve any tuning or scaling of the value of $r(t, M)$, as is necessary with the SAL or 2AL techniques.

\subsection{D settling of a particle in a rectangular tank}

The sedimentation of a spherical particle in a square section tank is considered. This test case corresponds to the experiments of Mordant and Pinton [26] where a rigid steel sphere 
of diameter $8 \times 10^{-4} \mathrm{~m}$ and density $7710 \mathrm{~kg} \cdot \mathrm{m}^{-3}$ is initially released without initial velocity. The particle is released into a liquid whose density and viscosity are $1000 \mathrm{~kg} \cdot \mathrm{m}^{-3}$ and $8.9 \times 10^{-4}$ Pa.s respectively. The calculation domain is considered unbounded as the radial containment is less than $10^{-3}$. The Reynolds number based on the particle diameter and its settling velocity is 280 and the corresponding Stokes number is 240.

In this section, several simulations are leaded on a $50 \times 50 \times 800$ grid associated to dimensions $0.004 m \times 0.004 m \times 0.064 m$ in Cartesian coordinates. The numerical domain is smaller than the experimental one. However, the magnitude of the Reynolds and Stokes numbers allows a small effect of choosing a numerical containment of 0.2 artificially higher than the experimental one to be evaluated.

The vertical component of the particle velocity is first investigated with the SAL, $2 \mathrm{AL}$ and 3AL methods (see figure 9). It is observed that the 2AL and 3AL approaches accurately predict the experimental measurements. On the contrary, the SAL method involves strong discrepancies with the measurements [26]. As it has been demonstrated in the work of Vincent et al. [49], a constant $r(t, M)$ and the resulting augmented Lagrangian penalty term alter dramatically the conditionning of the linear system in one of the fluids. The resulting simulations are not physically pertinent. Examples of velocity magnitudes and vectors, obtained with the SAL and 3AL methods at $t=0.15 \mathrm{~s}$, are shown in figure 10 in a vertical slice intersecting the particle center. The flow behind and in the rear of the particle is different. The toroidal velocity field, which is experimentally observed near the particle [26] and with the 3AL approach, is not recovered with the SAL method. The magnitude of the resulting velocities is $50 \%$ underestimated with the SAL method and the flow is not relevant in this case. The numerical overcost induced by the $3 \mathrm{AL}$ method is less than $1 \%$ of the time required to solve the Navier-Stokes equations and the extra memory requirements are negligible. 


\subsection{Free fall of a dense cylinder}

The purpose of this case is to demonstrate the ability of the 3AL method to deal with extreme cases and to compare this method with a classic time-splitting approach [21]. A cylindrical object of diameter $d=0.0125$, centered at $(0.05,0.175)$, is initially dropped in a rectangular cavity full of air whose dimensions are $[-0.05 ; 0.05] \times[-0.1 ; 0.1]$. The physical properties of air and solid media are the following: $\rho_{\text {air }}=1.1768 \mathrm{~kg} \cdot \mathrm{m}^{-3}, \rho_{\text {solid }}=10^{5} \mathrm{~kg} \cdot \mathrm{m}^{-3}$, $\mu_{\text {air }}=1.85 \times 10^{-5}$ Pa.s, $\mu_{\text {solid }}=10^{5}$ Pa.s. The interest of this case comes from consideration of high density and viscosity ratio. If it is assumed that the air has no effect on the fall of the solid, an analytical solution of the solid velocity $V$ can be easily found : $V=-g t$.

The behavior of the 3AL method is first investigated. The case is simulated on 5 meshes $(50 \times 100,100 \times 200200 \times 400,400 \times 800$ and $800 \times 1600$ points $)$. The time step is constant and equal to $6.25 \times 10^{-5} \mathrm{~s}$. The phase function $C$, which locates the dense cylinder, is advected with a VOF-PLIC method [7] and can induce deformations of cylinder shape. Another interest of this test case is to evaluate the ability of the 3AL method, coupled with an Eulerian interface tracking method, to handle the motion of a rigid object. The figure 11 shows, after 2500 time iterations, the isosurface $C=0.5$ describing the shape of the cylinder and the vorticity magnitude. The space convergence is observed while the cylindrical aspect of the solid is very well kept since the $100 \times 200$ mesh. On a quantitative point of view, the asymptotic velocity of the cylinder after 2500 iterations is estimated thanks to the Richardson extrapolation [24]. A limit value of $-1.52819 \mathrm{~m} \cdot \mathrm{s}^{-1}$ is obtained and used as reference value. Compared to the analytical invicid solution equal to $1.5328125 \mathrm{~m} . \mathrm{s}^{-1}$ for $t=0.15625 \mathrm{~s}$, the asymptotic numerical solution is in good agreement, even if the two solutions cannot be directly compared as the air medium is taken into account in our simulations. The figure 12 shows the relative error of the velocity. A regular convergence order of 0.8 is obtained. The second-order of the 3AL method is overridden by the error of the VOF-PLIC method and the estimate of the fall velocity by the discrete integral of the vertical velocities such that $C \geq 0.5$. Concerning the time convergence order, a $200 \times 400$ grid and seven time steps, 
i.e. from $4 \times 10^{-4} s$ to $0.625 \times 10^{-5} s$ by successive divisions by 2 , have been considered. The solutions of the velocity are taken at $t=0.78 \mathrm{~s}$. A Richardson extrapolation have provided an asymptotic value of the velocity of $-0.766807 \mathrm{~m} . \mathrm{s}^{-1}$. The evolution of the relative error according to the time step is described in table 3. The convergence order is irregular, with a mean value of 1.21 . The simulation on a $200 \times 400$ grid has been also performed with a time-splitting approach [21]. It has been observed that except for the smaller time step, i.e. $0.625 \times 10^{-5} s$, the simulations failed after several hundreds of time iterations due to the large divergence level of the prediction step. This step was discretized implicitly as explained in section 2.3. The correction step was not able to recover a correct divergence level after a certain time. For the smallest time step, the fall velocity admits a value of $-0.7704 m . s^{-1}$ with the projection approach, which is close to the value of $-0.7668 m . s^{-1}$ obtained with the 3AL method. The main interest of the augmented Lagrangian techniques is to allow the use of much larger time steps than with the time-splitting approaches, even for difficult cases such as the present case.

\section{Three-dimensional simulation of hydroplaning flows}

\subsection{Description of the problem}

The three-dimensional air-water flow interacting with a tire is considered for a tire rotation velocity of $46.3 \mathrm{rad} . \mathrm{s}^{-1}$ and a corresponding translational velocity of the road of $13.89 \mathrm{~m} . \mathrm{s}^{-1}$. The tire is a fictitious domain of imposed velocity which is accounted for into the calculation grid by means of penalty terms imposing the velocity in all the tire zone. As presented in figure 13, three tire geometries, called tires $T 1, T 2$ and $T 3$, are considered, in order to evaluate the effect of the tire patterns on the flow-structure interaction and resulting forces exerted on the tire during hydroplaning. The tires are shown as they are really projected onto the simulation grid. It is observed that only their bottom part is considered in the simulation. On the road, the tire is in contact with the bottom boundary of the simulation 
domain (empty parts of the tire surface in figure 13). It can be pointed that the tire structure is not accurately described in the upper part of the calculation domain, due to coarsening of the grid in this zone. However, the two-phase flow does not provide important features in this part of the simulation, so its influence on the hydroplaning motion can be solved in a coarser manner. A mixed scale LES model has been used to account of the subgrid turbulent scales. The interfacial scales are also under resolved in the wake of the tire. However, we have decided to take a coarser grid in this zone as it has no impact on the force calculation. At each time step, the movement of the tire structures, as well as the deformation of the tire, are calculated thanks to a home-made software by MICHELIN, which provides the triangular surface elements defining the tire topology. Consequently, the effect of the flow on the tire deformation are not currently taken into account. The tire deformations are principally due to the force exerted by the vehicle.

The fluid characteristics are $1000 \mathrm{~kg} \cdot \mathrm{m}^{-3}$ and $1.1768 \mathrm{~kg} \cdot \mathrm{m}^{-3}$ for the densities of water and air and $10^{-3}$ Pa.s and $1.85 \times 10^{-5}$ Pa.s for the dynamic viscosities in water and air respectively. The surface tension coefficient $\sigma$ between water and air is assumed to be equal to $0.075 N . \mathrm{m}^{-1}$. Initially, a water layer lays upward onto the road with a height of $8 \mathrm{~mm}$ on the total width of the road. The Reynolds number based on the initial water layer laying on the road and the road velocity is equal to $1.11 \times 10^{5}$ while the corresponding Weber number is $2.06 \times 10^{4}$. These two dimensionless numbers allow us to deduce that the flow is turbulent and that the surface tension effects are negligible in the inertial zones where the fluid-structure interaction reaches the maximum constraints.

The simulation grid is exponential, with a refined area in the zone where the tire is in contact with a road. The total number of cells in each directions is $270 \times 110 \times 80$ for the finest mesh, and $210 \times 95 \times 70$ and $150 \times 80 \times 60$ for two coarser grids used to study the spatial convergence. The size of the cells in the refined zone is $1 \mathrm{~mm}$ in each direction, while the 
main dimensions of the simulation domain are $1.4 m \times 0.291 \mathrm{~m} \times 0.6 \mathrm{~m}$. The grid structure in vertical and horizontal slice views is presented in figure 14. All the simulations are computed on the same grid which is the finest grid available for a sequential calculation. As discussed later, preliminary studies with coarser grids have shown that the force calculation were of the same order in terms of amplitude and frequency. The calculation time step is chosen constant and equal to $10^{-4} s$.

\subsection{Numerical results}

Figure 15 shows the convergence of the solver residual with $\mathcal{K}=1$ for two configurations at the time iteration 400. The first configuration uses 3 iterations of $3 \mathrm{AL}$ (minimization procedure) and 20 iterations of BiCG-Stab II and reaches a residual of $2.6 \times 10^{-4}$. For each 3AL iterations, the residual is decreased by one order of magnitude, from approximatively $10^{-2}$ to $10^{-4}$. The second more resolved configuration involves 4 iterations of $3 \mathrm{AL}$ (minimization procedure) and 30 iterations of BiCG-Stab II and reaches a residual of $1.4 \times 10^{-4}$. The total number of iterations is twice as for the first configuration and divides the final residual by only two. As is well known concerning the behavior of the iterative solvers, the decreasing of the residual slows down with the number of iterations. The same occurs with the number of iterations of the 3AL. A saturation is clearly observed when the number of iterations (solver or 3AL [18]) increases. The first configuration seems to be a good compromise between the solving quality and the computational cost. Concerning the physical meaning of the simulations, no macroscopic differences have been observed between the two solver configurations on the chosen grid. The same occurred for the $L^{2}$ norm of the divergence. The table 4 sums up the residual and divergence for the two configurations for each 3AL minimization iteration. It is observed that the divergence decreases slowly with the chosen $3 \mathrm{AL}$ parameters $\mathcal{K}=1$. The divergence can be reduced to almost computer error by using a projection step, for example the vectorial projection proposed in [18]. In order to save computational time, we choose to keep a relatively high divergence. The macroscopic physical aspect of the flow 
(and by consequence the force calculation on the obstacle) were not significantly improved by smaller values of divergence.

The same tests have been performed with $\mathcal{K}=100$ as described in figure 16 and table 5. As expected, the larger $\mathcal{K}$ is, the stronger the constraint is penalized (resulting a lower divergence) and the larger the residual is. With $\mathcal{K}=100$, the divergence is divided by 5 whereas the residual is multiplied by more than 50 . Hence, a parameter $\mathcal{K}=1$ is used in the rest of the paper. More generally, this behavior of the AL methods can be generalized to any variation of $\mathcal{K}$.

Concerning the computational time, a sequential version of our code has been used on one core of a Xeon Harpertown $3 \mathrm{Ghz}$ with 8 Go of RAM. As very specific ad hoc routines have been used, such as the generation of the mesh of the tire or the calculation of the surface forces, the parallel version of the code has not been used. Even if the number of total BiCG-Stab II iterations is multiplied by two between the configurations 1 and 2, 100 time iterations have been computed in 23 hours for the configuration 1 and in 29 hours for the configuration 2. In fact, 6 hours are used in the solver step for the configuration 1 and 12 hours for the configuration 2. Most of the rest of the calculation time is spent for generating and projecting the tire Lagrangian surface onto the Eulerian calculation grid. The cost of this operation can be divided by ten by using an octree data structure for the Eulerian-Lagrangian projection [1].

\subsection{Study of three-dimensional flows}

The three-dimensional unsteady two-phase flow occurring when a water-air free surface hits and interacts with a tire has never been studied numerically with a full unsteady description of the two-phase motion and the corresponding efforts exerted on the tire. The typical flow structures that are observed when the tire geometry changes are presented in figure 17 . The free-surface is represented by the iso-surface $C_{1}=0.5$. The macroscopic deformation of the free surface is observed to be periodic and therefore always evolves during time. On 
the contrary, unsteady small-scale 3D two-phase flow structures are noticed in the wake of the tire due to the development of turbulence. However, in figure 17, the simulations are considered after the flow has reached a stabilized state, i.e. the global shape of the free surface does not evolve during time on a macroscopic point of view. Whatever the type of tire topology, the macroscopic flow structure is almost the same. A V-like free surface form develops downstream the tire, a lot of water droplets are generated in the vicinity of the rotating obstacle and a pressure peak is created on the forward face of the tire near the road. The pressure field projected onto the tire surface is described in figure 18. The flow is observed with a bottom view perpendicular to the road. The simulated values of the maximum pressure on the tire are in the range $8 \times 10^{4}$ to $1 \times 10^{5} \mathrm{~Pa}$. These values are in good agreement with the experimental measurements of MICHELIN and the theoretical predictions provided by equation 1 :

$$
P_{h}=K V_{v}^{2}=96466 P a
$$

where the constant $K$ is equal to 500 and the velocity of the vehicle $V_{v}$ is $13.89 \mathrm{~m} . \mathrm{s}^{-1}$. On a two-phase point of view, it is observed that air tubes are generated when the water touches the tire on the side and in the wake of the obstacle. Under surface tension and shearing effects, these gas tubes break and generate bubbles and droplets, as can be observed in hydroplaning experiments.

\subsection{Analysis of forces exerted on a tire by water}

In this section, the vertical component of the normal pressure force $F_{n}^{p}$ exerted by the twophase flow on the tire is first studied. The figure (19) shows the convergence of $F_{n}^{p}$ for the three meshes presented previously. The results for the $270 \times 110 \times 80$ grid seems to be close to the convergence results. It justifies the use of this mesh.

The positive and negative contributions of $F_{n}^{p}$, defined as $F_{n}^{+}$and $F_{n}^{-}$, are introduced in 
order to estimate and discriminate the tire structure effect on the flow-structure interaction. The behavior of $F_{n}^{+}$and $F_{n}^{-}$is proposed in figure 20. For the three tires, the positive contribution of the vertical component of force $F_{n}^{p}$ is 7 to 8 orders of magnitude higher than the negative part $F_{n}^{-}$. This observation illustrates the hydroplaning configuration of the fluid-structure interaction considered in this work, i.e. the positive vertical component of the force exerted on the tire is larger than the negative one, involving hydroplaning if its magnitude is larger than the weight of the considered vehicle.

The time evolution of $F_{n}^{+}$admits a similar characteristic behavior for T1, T2 and T3. For $0 s \leqslant t \leqslant 0.01 s, F_{n}^{+}$increases until a maximum value between $800 N$ and $900 N$. This time interval corresponds to time required for the incident water layer to wet the tire surface near the road. For $0.01 s \leqslant t \leqslant 0.02 s$, the positive part of the vertical force component decreases, corresponding to the obtention of an almost equilibrium state between the incident water flow and the tire and road dynamics. After this time, the effort reaches an average asymptotic value included in the range $750 \mathrm{~N}$ to $850 \mathrm{~N}$. The main difference between the flat and structured tires is observed in the asymptotic region, for which the vertical force exerted by the two-phase flow is constant for $T 1$ (flat tire) whereas regular oscillations are numerically measured for $T 2$ and $T 3$, which are characteristic of the tire structure.

The discrimination of the tire is now investigated by considering the evolution of the total vertical force $F_{n}^{p}$ during time. These results are presented in figure 21 . It is observed that building a structure on a tire reduces by $20 \%$ the effort exerted by water on the tire, as observed experimentally by tire manufacturers. As for $F_{n}^{+}$and $F_{n}^{-}$, after the fluid-structure interaction has reach a stabilized state for $t \geq 0.02 s, F_{n}^{p}$ linearly increases over time. This results is the correlated increase of the ambient pressure in the calculation domain. No boundary conditions are required on the pressure with the AL approach, so the absolute pressure in the domain is free to evolve in the case of open boundary conditions. Only the 
pressure gradient is required in the momentum equation and wall force should not be affected by the pressure level. However, it is true if the force integration is performed on a closed surface. As the tire is cut by the domain, the force is not entirely computed and is slightly sensitive to the global pressure level. The real effort exerted on the tire after $t=0.02 \mathrm{~s}$ no more increases. This could be verified numerically by subtracting the ambient pressure to the pressure calculated locally. In this case, the pressure in the air should be negligible almost everywhere.

A difficulty would lie in the choice of the ambient pressure in our simulations. However, the important feature here is the classification of the forces resulting from the fluid-structure interaction according to the tire structure, which is nicely established by the simulations. Contrary to the classification brought by $F_{n}^{+}$, the $F_{n}^{p}$ curve demonstrates that the tire $T 3$ involves a $5 \%$ in average lower effort than T2. For these two tire geometries, the main difference lies in the opportunity provided by $T 3$, due to its shape design, to exert a negative vertical effort which compensate the value of $F_{n}^{+}$and allows $F_{n}^{p}$ to be lower for $T 3$ than for $T 2$.

A last interesting parameter can be extracted from the simulated efforts: the characteristic frequencies arising when the tires are patterned. It is recalled and observed in the simulations that no typical periodic variations are observed for a flat tire, as expected. The best variable allowing to measure the characteristic time variations of efforts is $F_{n}^{+}$, as observed in figure 20. A zoom of the positive vertical contributions of $F_{n}^{p}$ is presented in figure 22. The analysis of the $F_{n}^{+}$signals allows the characteristic frequencies $f_{T 2}$ and $f_{T 3}$ of tires $T 2$ and $T 3$ to be extracted:

$$
\left\{\begin{array}{c}
f_{T 2}=25 / 0.089=280 H z \\
f_{T 3}=32 / 0.091=351 H z
\end{array}\right.
$$

It can be given an attempt to correlate $f_{T 2}$ and $f_{T 3}$ to the typical tire structures presented in figure 23. A frequency can be estimated by dividing the relative velocity of the road $V_{v}=$ 
$13.89 \mathrm{~m} / \mathrm{s}$ by a characteristic distance. If the vertical distance between the tire structures is used, it can be shown that

$$
\left\{\begin{array}{l}
f_{T 2}=13.89 / 0.048=289 H z \\
f_{T 3}=13.89 / 0.040=347 H z
\end{array}\right.
$$

As a conclusion, it has been demonstrated that the fluctuations observed on the time evolution of the efforts are directly dependent on the size of the larger structure of the considered tire.

To synthesize the results, the three-dimensional two-phase flow structure interacting with the tire have been clearly simulated. The corresponding efforts exerted on the tire have been compared for three different tires. A classification of the tire topologies has been proposed with respect to the magnitude of the total vertical normal forces, i.e. the flat tire involves a $20 \%$ higher effort than the structured tires. This demonstrates that using a structured tire clearly reduces the vertical force exerted on the tire and that in this case, the hydroplaning will occur for higher road velocities. These results demonstrate the importance of the design of tire which allow the hydrodynamic pressure in front of the tire [38] to be minimized. Eventually, characteristic frequencies of structured tires are clearly observed. They are related to the size of the larger tire structure.

A parametric study has been carried out concerning the refinement of the grid and the solver parameters. The grid used in the present work, $270 \times 110 \times 80$, has the maximum number of cells available for a sequential calculation. Simulation with coarser grids, $150 \times$ $110 \times 80$ for example, have provided differences on the surface forces of maximum $20 \%$ 


\section{Concluding remarks}

A two-phase flow model dedicated to the simulation of hydroplaning, based on a generalized formulation of the Navier-Stokes equations for unsteady free-surface flows, has been presented. The interaction between the air-water flow and the tire is modeled thanks to fictitious domains. On a numerical point of view, penalty methods are proposed to take into account the fluid-structure interaction on fixed grids with artificial sub-domains being merged into. In addition, an original algebraic augmented Lagrangian method has been proposed for an accurate treatment of the velocity-pressure coupling which is second-order in space accurate intrinsically. We have obtained a second-order accuracy for the thermally driven cavity case, while first order have been measured in the framework of two-phase flows. It can be explained by the lower orders of the phase tracking methods. However, it has been demonstrated that larger time steps can be used with AL methods than with a time-splitting approach.

The two-phase flow model has been successfully validated on the collapse of a water column and in the case of a particle settling in a rectangular tank. The experimental measurements available concerning these two test cases are accurately recovered by the simulations. In addition, a numerical study is provided on augmented Lagrangian methods that demonstrates the superiority of the algebraic adaptive augmented Lagrangian (3AL) method over other existing methods (SAL, 2AL): more physically relevant results are obtained while the numerical cost is decreased.

Concerning hydroplaning simulations and the simulation of three-dimensional two-phase flows interacting with moving obstacles, it has been demonstrated that the 3AL method and penalty techniques can be a usefull tool which is easy to implement for engineering applications while using the real object shape on fixed structured grids. The estimate of surface constraints exerted by water on various patterned tires has provided new results and 
explanations allowing to discriminate the tires according to their typical pattern shape.

\section{Acknowledgements}

The authors thank the MICHELIN group for its financial support and the Aquitaine Regional Council for the financial support dedicated to a 256-processor cluster investment, located in the TREFLE laboratory. This work was also granted access to the HPC resources of CCRT, CINES and IDRIS by GENCI (Grand Equipement National de Calcul Intensif) under reference number x2009026115. 


\section{References}

[1] Sarthou A., Vincent S., and Caltagirone J.-P. A second-order curvilinear to cartesian transformation of immersed interfaces and boundaries. application to fictitious domains and multiphase flows. Hal preprint: http://hal.archives-ouvertes.fr/hal-00479705, 2010.

[2] Sarthou A., Vincent S., Caltagirone J.-P., and Angot P. Eulerian-lagrangian grid coupling and penalty methods for the simulation of multiphase flows interacting with complex objects. Int. J. Numer. Meth. Fluids, 56:1093-1099, 2008.

[3] Sarthou A., Vincent S., Angot P., and Caltagirone J.-P. The sub-mesh-penalty method. Finite Volumes for Complex Applications V, pages 633-640, 2008.

[4] Aksenov A.A., Dyadkin A., and Gudzovsky A.V. Numerical simulation of car tire aquaplaning. ECCOMAS 96, Paris, 1996.

[5] Aksenov A.A. and Gudzovsky A.V. The software flowvision for study of air flows, heat and mass transfer by numerical modelling methods. Proc. of the Third Forum of Association of Engineers for Heating, Ventilation, Air-Conditioning, Heat Supply and Building Thermal Physics, 22-25 Aug., Moscow (in Russian), 1993.

[6] Lacanette D., Gosset A., Vincent S., Buchlin J.-M., and Arquis E. Macroscopic analysis of gas-jet wiping: Numerical simulation and experimental approach. Phys. Fluid, 18:1$15,2006$.

[7] Youngs D.L. Time-dependent multimaterial flow with large fluid distortion. Morton K.W. and Baines M.J. (editors), Numerical Methods for Fluid Dynamics, Academic Press, New-York, 1982.

[8] Labourasse E., Lacanette D., Toutant A., Lubin P., Vincent S., Lebaigue O., Caltagirone J.-P., and Sagaut P. Detailed comparisons of front-capturing methods for turbulent twophase flow simulations. Int. J. Mult. Flow, 33:1-39, 2007. 
[9] Bertrand F., Tanguy P.A., and Thibault F. A three-dimensional fictious domain method for incompressible fluid flow problems. Int. J. Numer. Meth. Fluids, 25:716-736, 1997.

[10] Pianet G., Vincent S., Leboi J., Caltagirone J.-P., and Anderhuber M.”. Simulating compressible gas bubbles with a smooth volume tracking 1-fluid method. International Journal of Multiphase Flow, 36(4):273 - 283, 2010.

[11] Uzawa H. Iterative method for concave programming. in K.J. Arrow, L/ Hurwicz, H. Uzawa, editors, Studies in linear and nonlinear programming, Stanford University Press. 1958.

[12] Van Der Vorst H.A. Bicg-stab: a fast and smoothly converging variant of bi-cg for the solution of nonsymmetric linear systems. SIAM Journal on Scientific and Statistical Computing, 13(2):631-644, 1992.

[13] Gustafsson I. On First and Second Order Symmetric Factorization Methods for the Solution of Elliptic Difference Equations. Research report, Department of Computer Sciences, Chalmers University of Technology and the University of Gateborg. 1978.

[14] Kataoka I. Local instant formulation of two-phase flow. Int. J. Multiphase Flow, $12(5): 745-758,1986$.

[15] Bardina J., Ferziger J.H., and Reynolds W.C. Improved subgrid scale models based on large eddy simulation. AIAA Paper, 80:1357, 1980.

[16] Larocque J., Rivière N., Vincent S., Reungoat D., Fauré J.-P., Héliot J.-P., and Caltagirone J.-P. Macroscopic analysis of a turbulent round liquid jet impinging on an air/water interface in a confined medium. Phys. Fluid, 21:065110-1-21, 2009.

[17] Caltagirone J.-P. On the fluid-porous interaction, application to the calculation of efforts exerted on an obstacle by a viscous fluid. C. R. Acad. Sci. Série IIb, 318:571-577, 1994. 
[18] Caltagirone J.-P. and Breil J. A vectorial projection method for solving the navier-stokes equations. C. R. Acad. Sci. Série IIb, 327:1179-1184, 1999.

[19] Delhaye J.M. Jump conditions and entropy sources in two-phase systems. local instant formulation. Int. J. Multiphase Flow, 1(3):395-409, 1974.

[20] Brackbill J.U., Kothe D.B., and Zemach C. A continuum method for modeling surface tension. J. Comput. Phys., 100(2):335-354, 1992.

[21] Goda K. A multistep technique with implicit difference schemes for calculating two- or three-dimensional cavity flows. J. Comput. Phys., 30:76-95, 1978.

[22] Khadra K., Angot P., Parneix S., and Caltagirone J.-P. Fictitious domain approach for numerical modelling of navier-stokes equations. Int. J. Numer. Meth. Fluid, 34:651-684, 2000.

[23] Masataka K. and Toshihiko O. Hydroplaning simulation using msc.dytran. MSC Publication.

[24] Richardson L.F. and Gaunt J.A. The deferred approach to the limit. Transactions of the Royal Society of London. Series A: Mathematical and Physical Sciences, 226:299-361, 1927.

[25] Fortin M. and Glowinski R. Augmented Lagrangian: application to the numerical solution of boundary value problems. Ed. North-Holland, Amsterdam. 1983.

[26] Mordant N. and Pinton J.-F. Velocity measurements of settling sphere. Eur. Phys. J. B, 18:343-352, 2000.

[27] Tunnicliffe N., Chesterton J., and Nancekivell N. The use of the gallaway formula for aquaplaning evaluation in new zealand. NZIHT Transit NZ 8 Annual Conference, 2006.

[28] Lebaigue O., Ducquennoy C., and Vincent S. Test-case no 1: Rise of a spherical cap bubble in a stagnant liquid (pn). Multiphase Sci. Tech., 6:1-4, 2004. 
[29] Schenk O. and Gärtner K. Solving unsymmetric sparse systems of linear equations with pardiso. Journal of Future Generation Computing Systems, 20:475-487, 2004.

[30] Le Quéré P. Accurate solutions to the square thermally driven cavity at high rayleigh number. Computers \& Fluids, 20(1):29 - 41, 1991.

[31] Lubin P., Vincent S., Abadie S., and Caltagirone J.-P. Three-dimensional large eddy simulation of air entrainment under plunging breaking waves. Coastal Eng., 53:631-655, 2006.

[32] Sagaut P. Large Eddy Simulation for incompressible flows - An introduction. Springer Verlag Berlin, 1998.

[33] Trontin P., Vincent S., J.-L Estivalezes, and J.-P. Caltagirone. Detailed comparisons of front-capturing methods for turbulent two-phase flow simulations. Int. J. Numer. Meth. Fluids, 56:1543-1549, 2008.

[34] Cho R., Lee H. W., Sohn J. S., Kim G. J., and Woo J. S. Numerical investigation of hydroplaning characteristics of three-dimensional patterned tire. Eur. J. Mech. A Solid, 25:914-926, 2006.

[35] Hestenes M. R. Multiplier and gradient methods. J. Optimization Theory and Applications, 4:330-320, 1969.

[36] Scardovelli R. and Zaleski S. Direct numerical simulation of free surface and interfacial flows. Ann. Rev. Fluid Mech., 31:567-603, 1999.

[37] Cabezón R.M., García-Senz D., and Relano A. A one-parameter family of interpolating kernels for smoothed particle hydrodynamics studies. J. Comput. Phys., 227:8523-8540, 2008 .

[38] Yeager R.W. and Tuttle J.L. Testing and analysis of tire hydroplaning. SAE Paper, 720471, 1972. 
[39] Koshizuka S. and Oka Y. Local instant formulation of two-phase flow. Nuclear Sci. Eng., 123(5):421-434, 1996.

[40] Osher S. and Fedkiw R. Level set methods: an overview and some recent results. J. Comput. Phys., 169:463-502, 2001.

[41] Shin S. and Juric D. Modeling three-dimensional multiphase flow using a level contour reconstruction method for front tracking without connectivity. J. Comput. Phys., 202:427-470, 2002.

[42] Vincent S., Balmigère G., Caruyer C., Meillot E., and Caltagirone J.-P. Contribution to the modeling of the interaction between a plasma flow and a liquid jet. Surf. Coating Tech., 203:2162-2171, 2009.

[43] Vincent S., Larocque J., Lacanette D., Toutant A., Lubin P., and Sagaut P. Numerical simulation of phase separation and a priori two-phase les filtering. Comput. Fluids, 37(7):898-906, 2008.

[44] Vincent S., Larocque J., Lubin P., Caltagirone J.-P., and Pianet G. Adaptive augmented lagrangian techniques for simulating unsteady multiphase flows. $6^{\text {th }}$ Int. Conference Multiphase Flow, Leipzig, Germany, July 9-13, 203, 2007.

[45] Vincent S. and Caltagirone J.-P. Efficient solving method for unsteady incompressible interfacial flow problems. Int. J. Numer. Meth. Fluids, 30(6):795-811, 1999.

[46] Vincent S. and Caltagirone J.-P. Numerical solving of incompressible navier-stokes equations using an original local multigrid refinement method. Comptes Rendus de l'Académie des Sciences - Series IIB - Mechanics-Physics-Astronomy, 328(1):73 - 80, 2000 .

[47] Vincent S. and Caltagirone J.-P. A one-cell local multigrid method for solving unsteady incompressible multiphase flows. J. Comput. Phys., 163(1):172-215, 2000. 
[48] Vincent S., Caltagirone J.-P., Lubin P., and Randrianarivelo T.N. An adaptative augmented lagrangian method for three-dimensional multimaterial flows. Comput. Fluids, 33(10):1273-1289, 2004.

[49] Vincent S., Randrianarivelo T.N., Pianet G., and Caltagirone J.-P. Local penalty methods for flows interacting with moving solids at high reynolds numbers. Comput. Fluids, 36(5):902-913, 2007.

[50] J. Smagorinsky. General circulation experiments with the primitive equations. i : The basic experiments. Month. Weath. Rev., 91:99-165, 1963.

[51] Rider W.J. and Kothe D. Stretching and tearing interface tracking methods. in the 12 ${ }^{\text {th }}$, AIAA CFD Conference, San Diego, June 20, page 1717, 1995. 


\begin{tabular}{rrrr}
\hline $\mathrm{Ra}$ & $10^{5}$ & $10^{6}$ & $10^{7}$ \\
\hline Le Quéré [30] & 4.521 & 8.8252 & 16.523 \\
Asymptotic solution & 4.521638 & 8.825208 & 16.5232 \\
Order & 1.9983 & 1.9974 & 1.9939 \\
\hline
\end{tabular}

Table 1: Thermally driven cavity - Reference Nusselt values of [30], asymptotic convergence values of the $N u$, and convergence orders for different $R a$ numbers.

\begin{tabular}{ccccc}
\hline Method & Number of BiCG-Stab II iterations & Residual & Divergence norm & CPU time (s) \\
\hline SAL & 5 & $3.2 \times 10^{-3}$ & $2.8 \times 10^{-3}$ & 86 \\
SAL & 10 & $1.0 \times 10^{-3}$ & $2.8 \times 10^{-3}$ & 124 \\
SAL & 50 & $2.7 \times 10^{-5}$ & $2.8 \times 10^{-3}$ & 438 \\
3AL & 5 & $4.0 \times 10^{-3}$ & $2.1 \times 10^{-2}$ & 96 \\
3AL & 10 & $9.4 \times 10^{-4}$ & $7.5 \times 10^{-3}$ & 141 \\
3AL & 50 & $3.5 \times 10^{-5}$ & $2.7 \times 10^{-3}$ & 439 \\
\hline
\end{tabular}

Table 2: Numerical performances of the augmented Lagrangian techniques for the collapse of a water column on a $400 \times 800$ grid.

\begin{tabular}{rrr}
\hline Time $(\mathrm{s})$ & Relative error & Order \\
\hline $4 \times 10^{-4}$ & $6.35 \times 10^{-4}$ & \\
$2 \times 10^{-4}$ & $2.20 \times 10^{-4}$ & 1.53 \\
$1 \times 10^{-4}$ & $1.38 \times 10^{-4}$ & 0.67 \\
$0.5 \times 10^{-4}$ & $4.34 \times 10^{-5}$ & 1.67 \\
$2.50 \times 10^{-5}$ & $3.48 \times 10^{-5}$ & 0.32 \\
$1.25 \times 10^{-5}$ & $1.0 \times 10^{-5}$ & 1.66 \\
$6.25 \times 10^{-6}$ & $2.6 \times 10^{-6}$ & 1.89 \\
\hline
\end{tabular}

Table 3: Relative errors and corresponding convergence orders on the fall velocities of a dense cylinder for various time steps - values obtained with the 3AL method. 


\begin{tabular}{rrrrr}
\hline Iteration & Residual config. 1 & Divergence config. 1 & Residual config. 2 & Divergence config. 2 \\
\hline 1 & $6.70 E-03$ & $6.90 E-02$ & $4.50 E-03$ & $4.40 E-02$ \\
2 & $1.00 E-03$ & $6.10 E-02$ & $8.80 E-04$ & $3.80 E-02$ \\
3 & $2.60 E-04$ & $5.30 E-02$ & $2.90 E-04$ & $3.50 E-02$ \\
4 & & & $1.30 E-04$ & $3.30 E-02$ \\
\hline
\end{tabular}

Table 4: Hydroplaning simulations - Residual and divergence for 3 iterations of 3AL and 20 iterations of BiCG-Stab II (config. 1) and for 4 iterations of $3 \mathrm{AL}$ and 30 iterations of BiCG-Stab II (config. 2). In step 6 of $3 \mathrm{AL}, \mathcal{K}=1$.

\begin{tabular}{rrrrr}
\hline Iteration & Residual config. 1 & Divergence config. 1 & Residual config. 2 & Divergence config. 2 \\
\hline 1 & $1.70 E-1$ & $1.10 E-02$ & $5.10 E-02$ & $1.10 E-02$ \\
2 & $5.00 E-02$ & $9.70 E-03$ & $2.00 E-02$ & $8.50 E-03$ \\
3 & $3.30 E-02$ & $9.40 E-03$ & $1.10 E-02$ & $8.50 E-03$ \\
4 & & & $6.60 E-03$ & $8.60 E-03$ \\
\hline
\end{tabular}

Table 5: Hydroplaning simulations - Residual and divergence for 3 iterations of 3AL and 20 iterations of BiCG-Stab II (config. 1) and for 4 iterations of 3AL and 30 iterations of BiCG-Stab II (config. 2). In step 6 of $3 \mathrm{AL}, \mathcal{K}=100$.

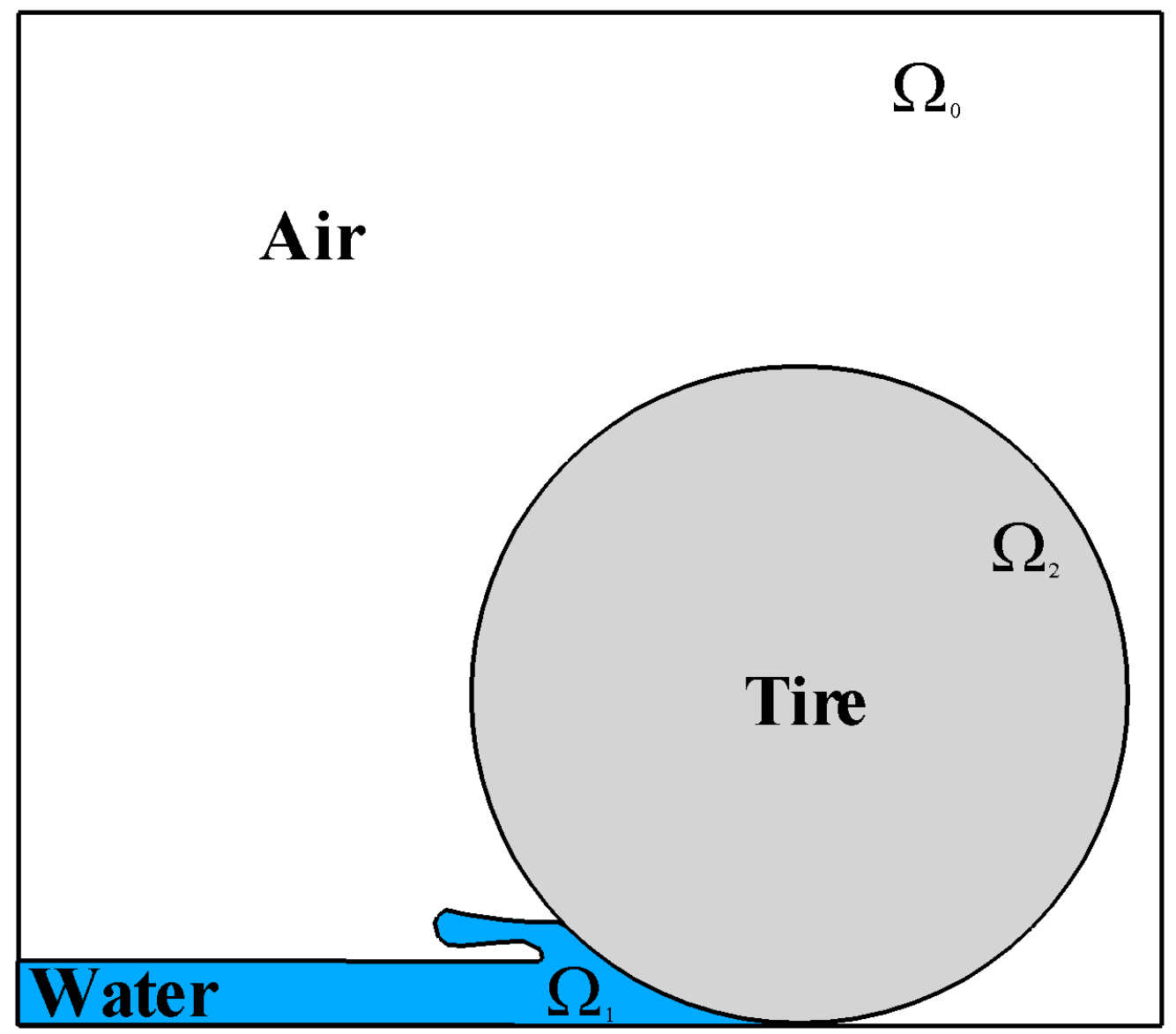

Figure 1: Definition sketch of the fictitious domain method for the hydroplaning flow. 


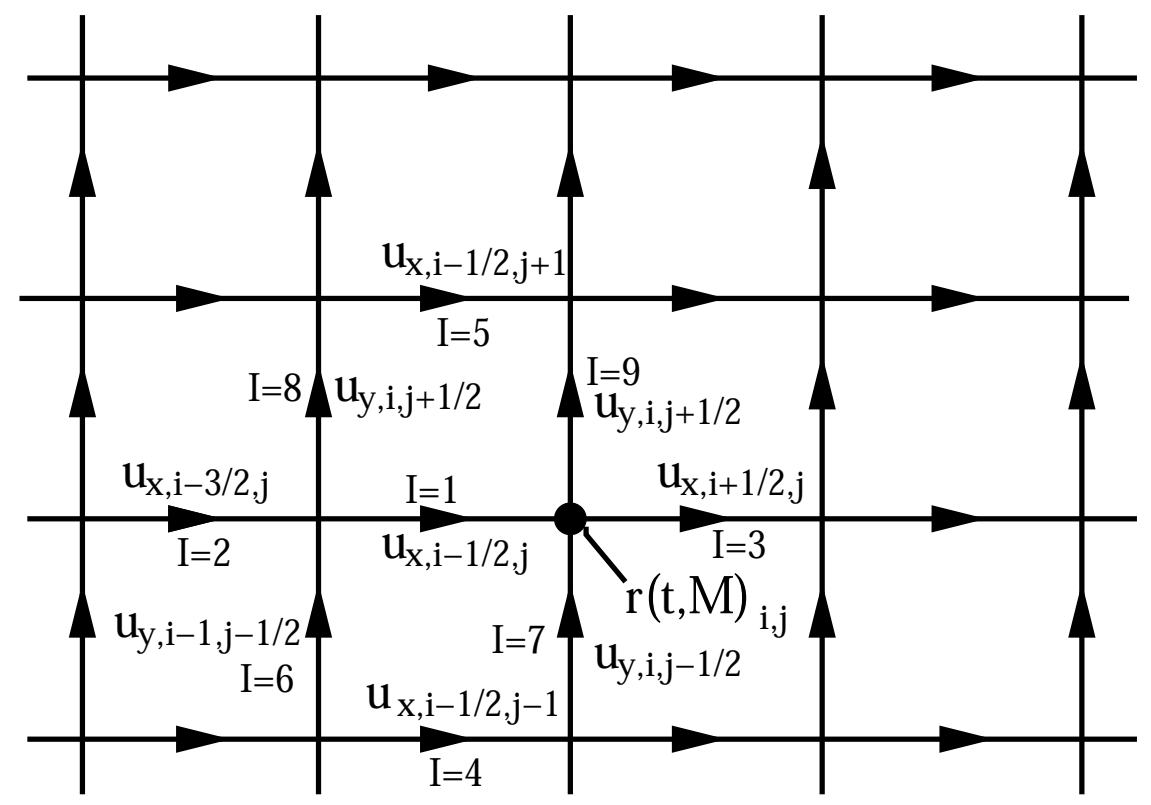

Figure 2: Typical distribution of discretization variables on a 2D fixed staggered Cartesian grid.

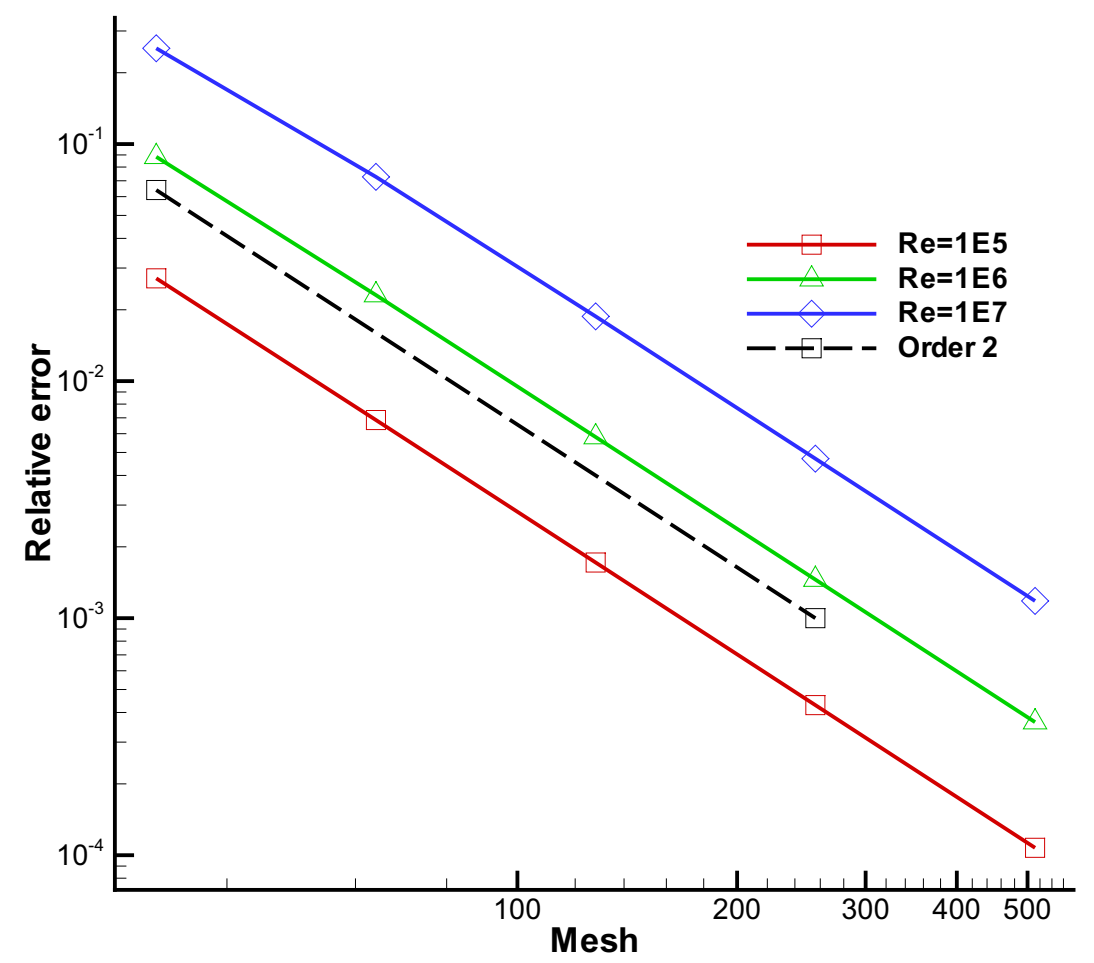

Figure 3: Spatial convergence of the relative error on the Nusselt number for the square thermally driven cavity. 


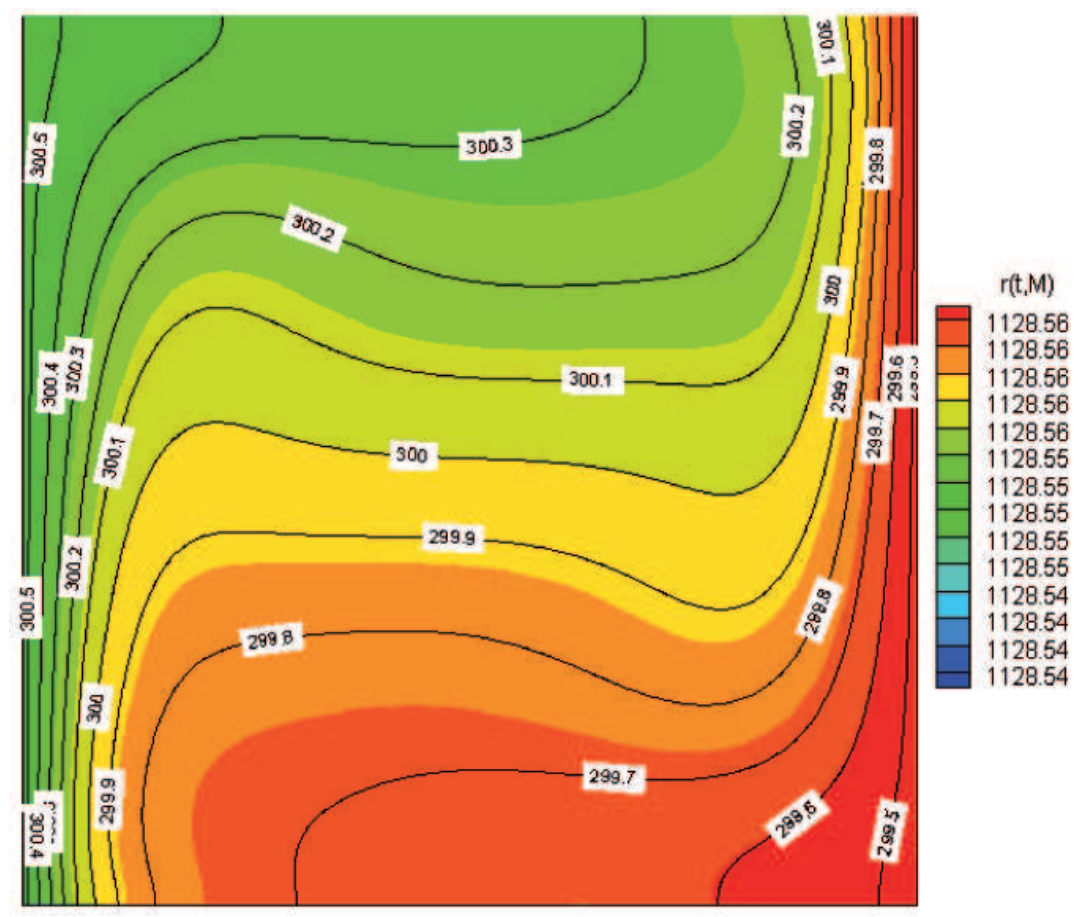

Figure 4: Spatial distribution of $r(t, M)$ (colors) and isolines of temperature for $R a=10^{5}$ on a $512 \times 512$ mesh. 


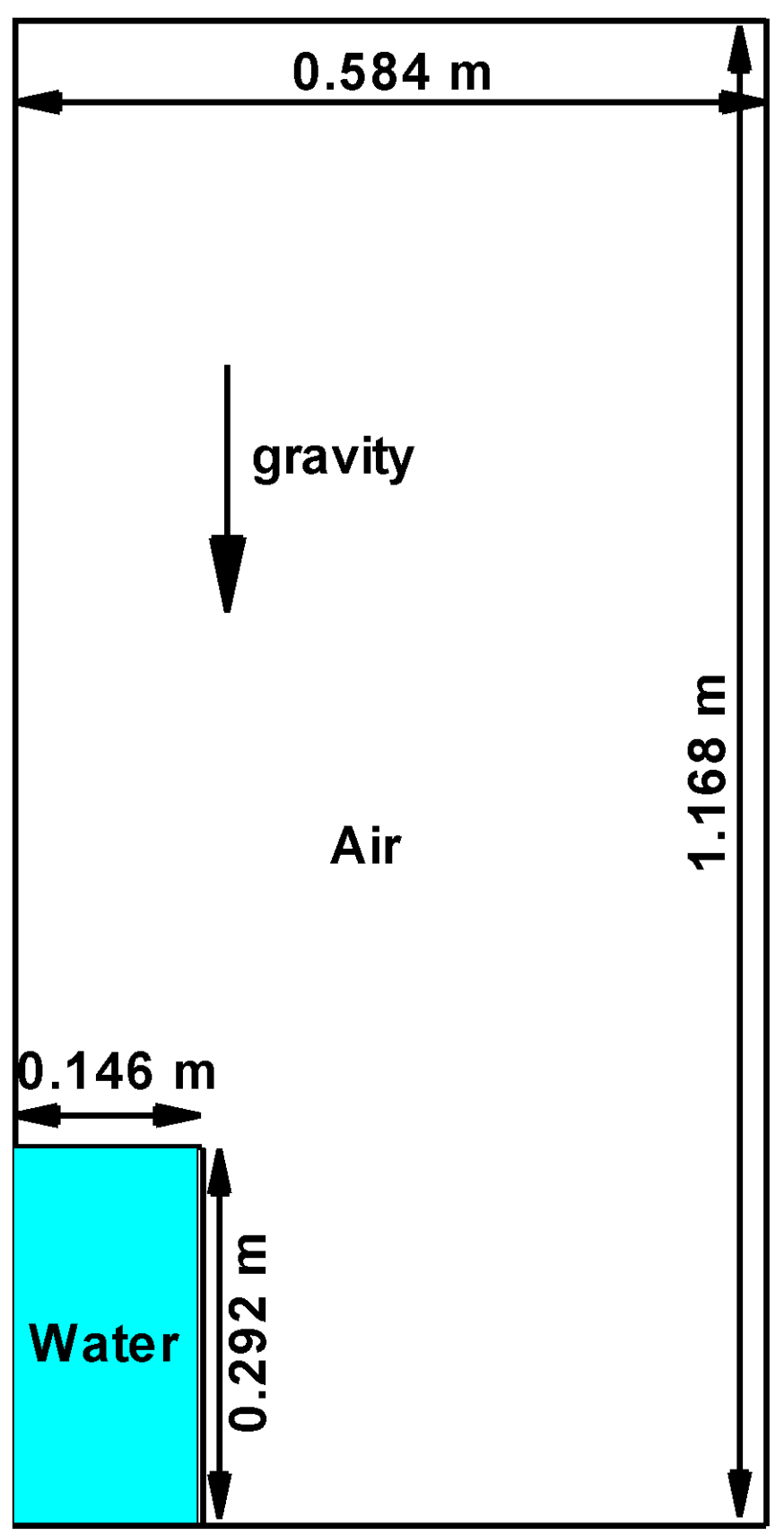

Figure 5: Definition sketch of the collapse of a water column in a square tank. 

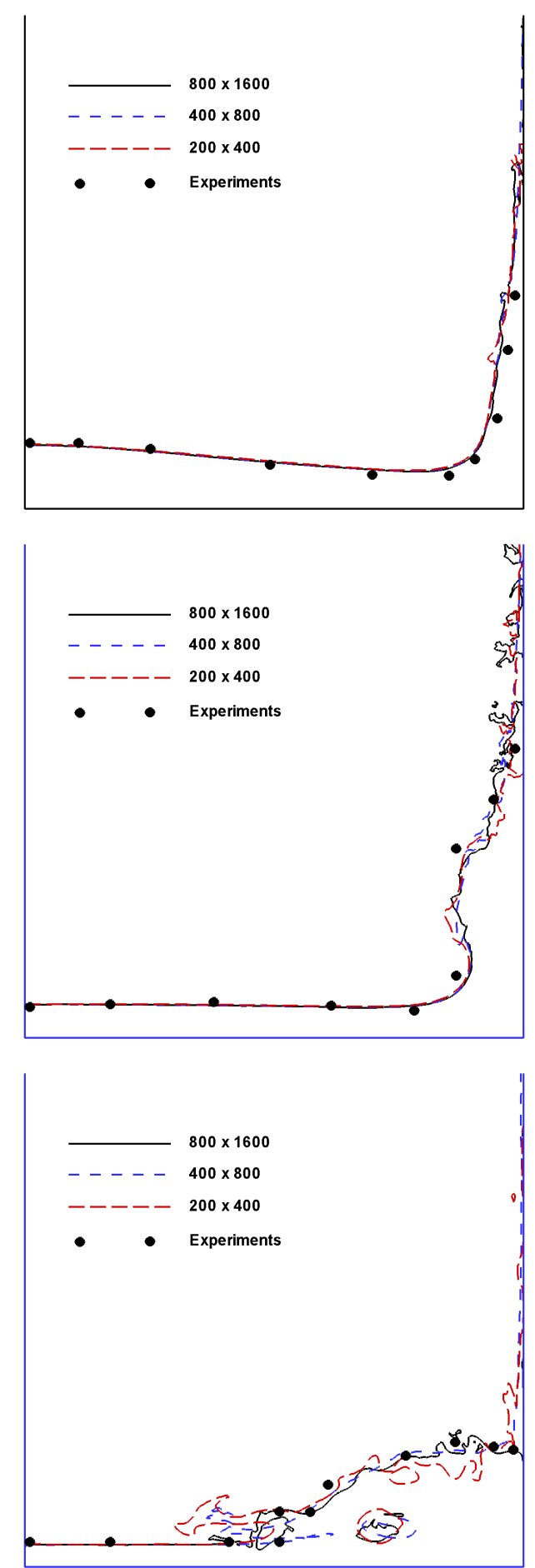

Figure 6: Simulation of the collapse of a water column - Convergence study of the free surface position for $t=0.4,0.6$ and $0.8 \mathrm{~s}$ with the $3 \mathrm{AL}$. 

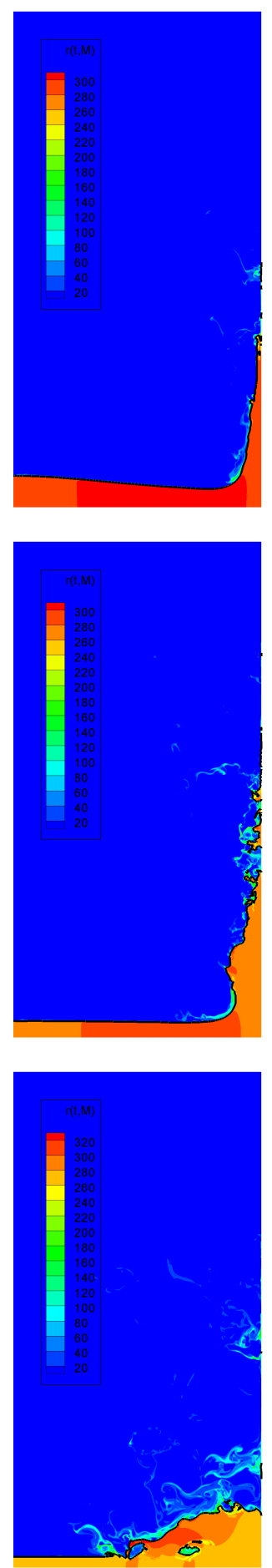

Figure 7: Simulation of the collapse of a water column on a $400 \times 800$ grid - Magnitude of the $3 \mathrm{AL}$ parameter (iso-contours) and free surface (black line) for $t=0.4,0.6 \mathrm{~s}$. and $0.8 \mathrm{~s}$. 


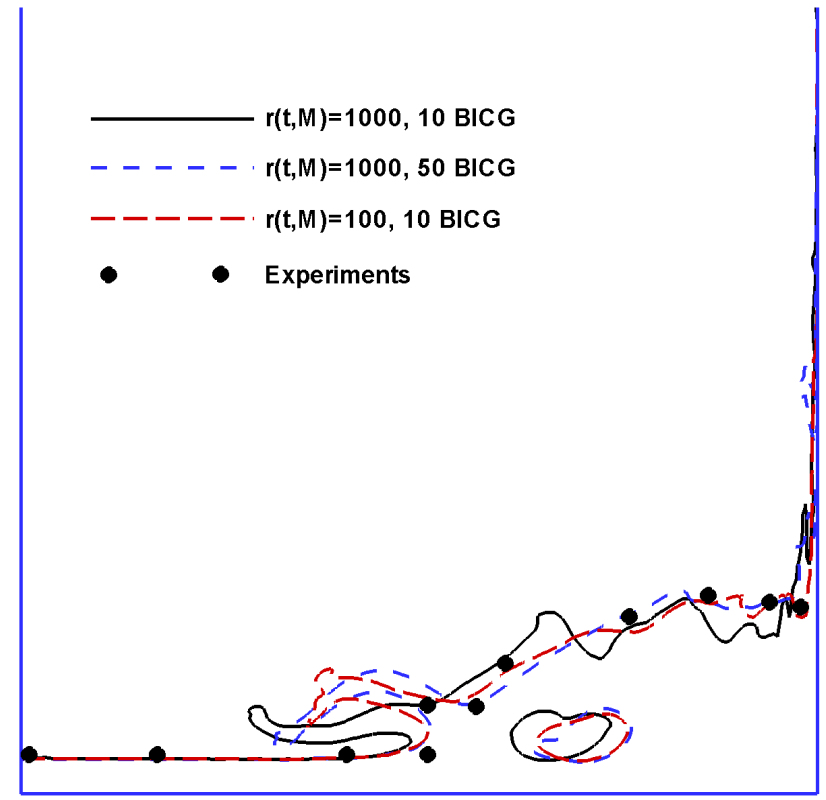

Figure 8: Simulation of the collapse of a water column - Comparison between several SAL simulations on a $400 \times 800$ grid at $t=0.8$ to experiments. 

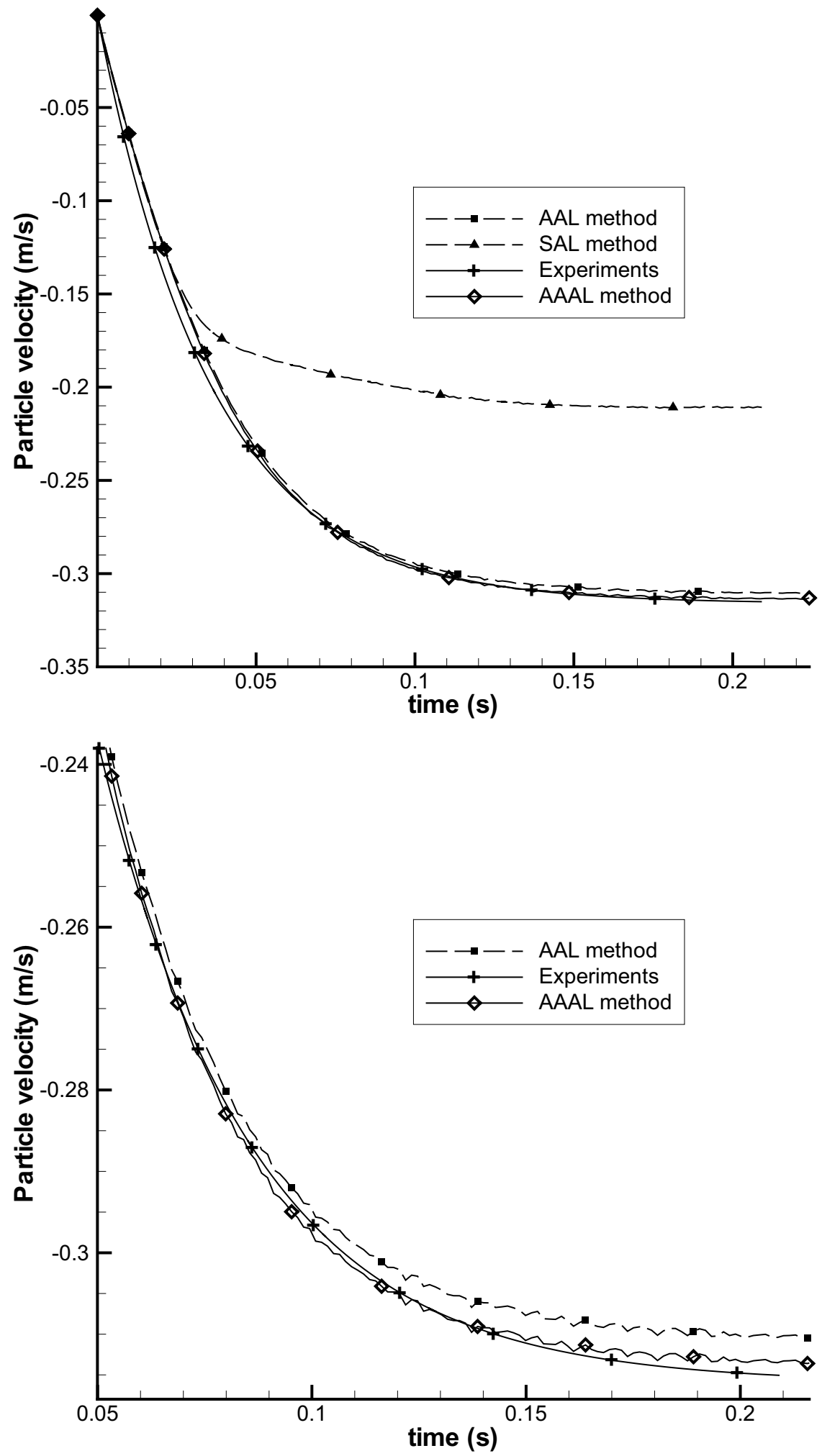

Figure 9: Simulation of the settling of a spherical particle at $R e=280$ - Evolution of the particle velocity during time - Comparison between SAL, 2AL, 3AL and experimental results (top) and zoom for the same comparison between $t=0.05 \mathrm{~s}$ and $t=0.025 \mathrm{~s}$ (bottom). 

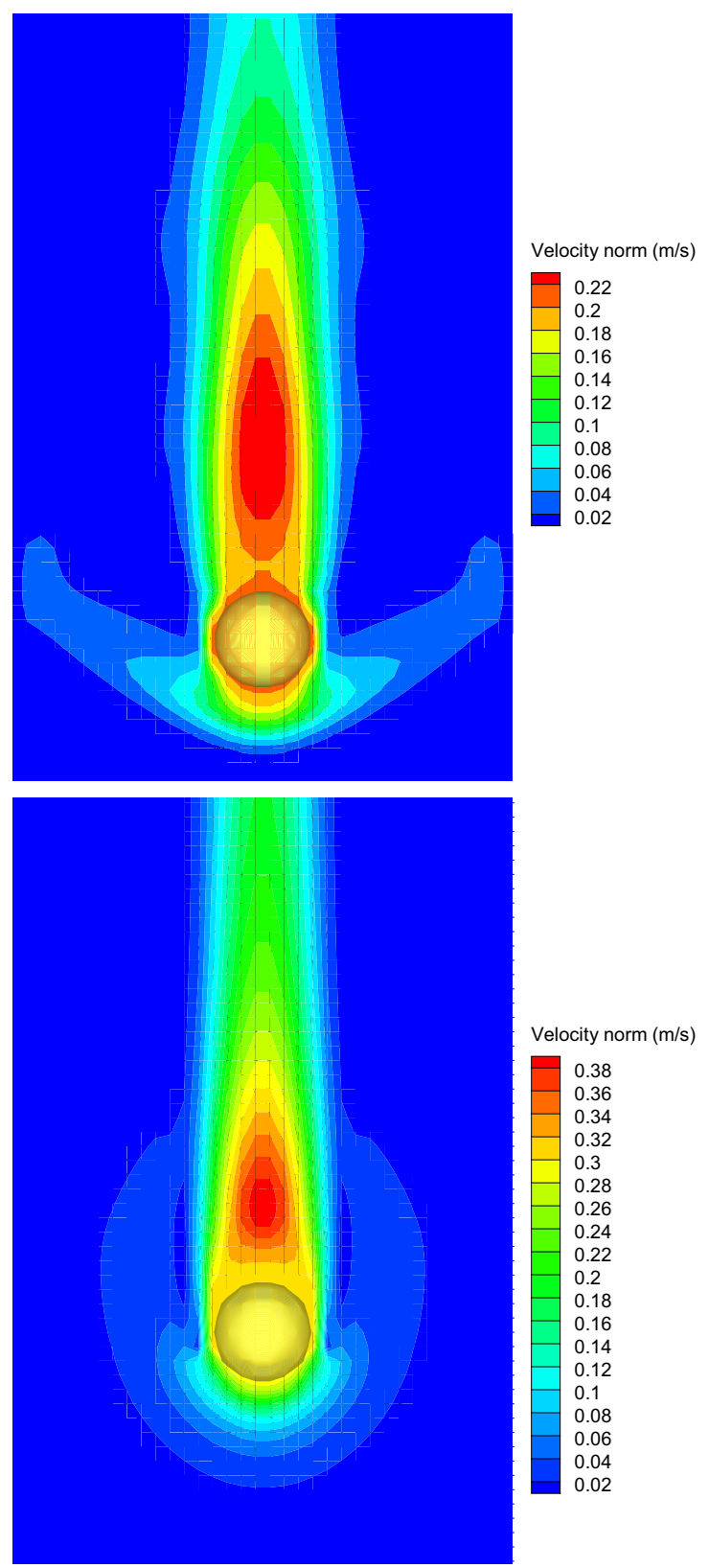

Figure 10: Simulation of the settling of a spherical particle at $R e=280$ - Isolines of vertical velocity norms in a vertical slice cutting the particle barycenter - Comparison between SAL (top) and 3AL (bottom) results. 

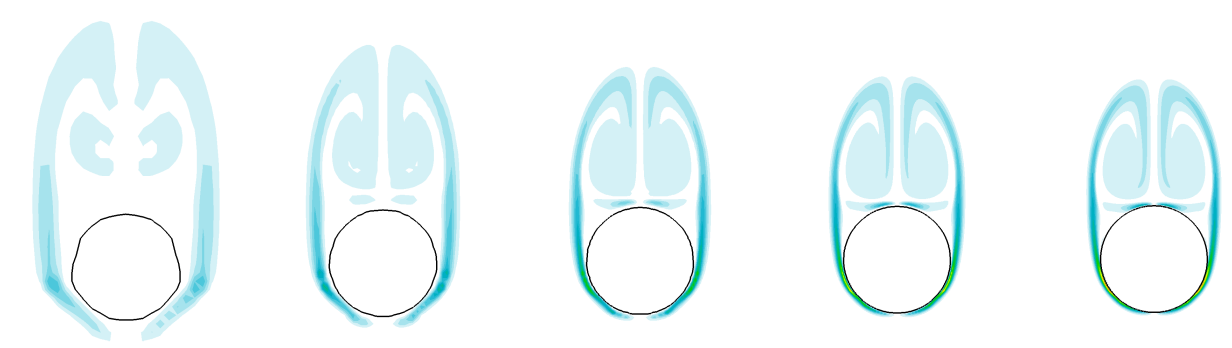

Figure 11: Vorticity magnitude and iso $C=0.5$ for the free fall of a dense cylinder with $50 \times 100,100 \times 200,200 \times 400,400 \times 800$ and $800 \times 1600$ meshes.

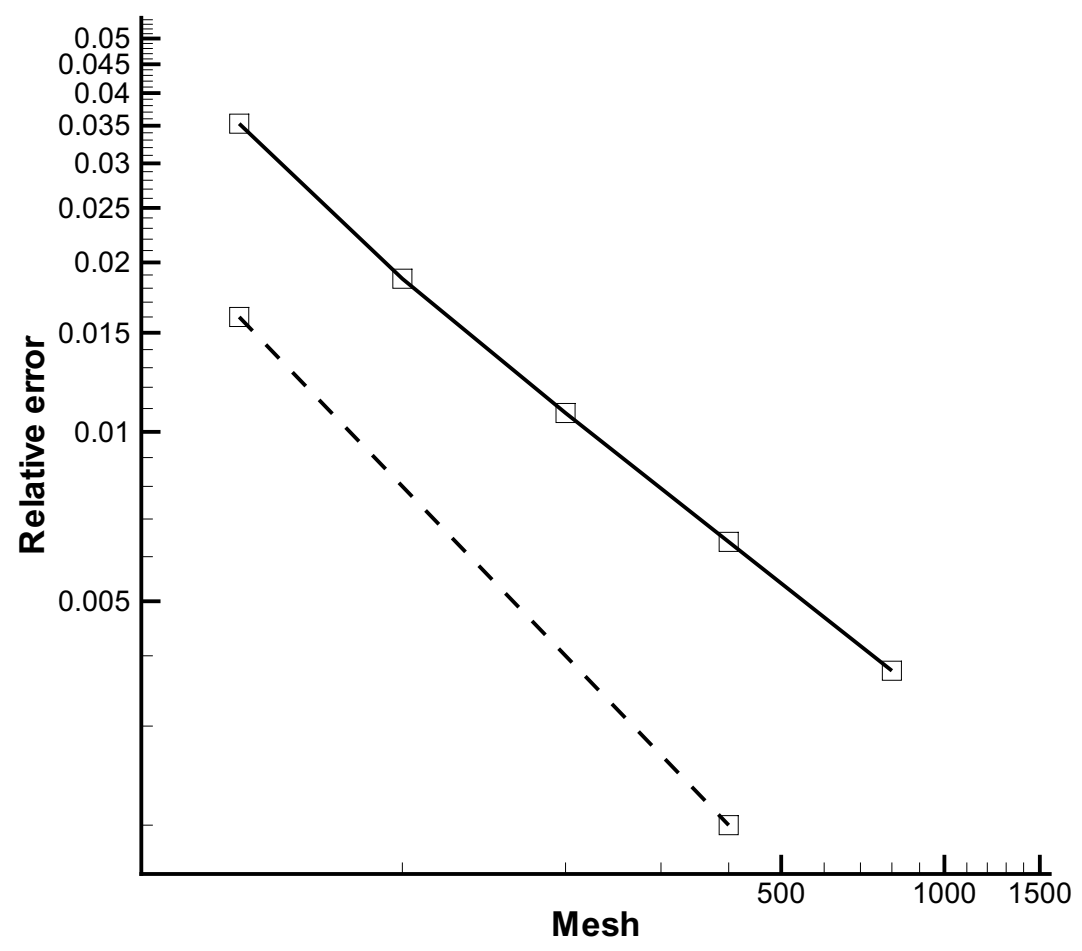

Figure 12: Convergence of the relative error on the fall velocity for the 3AL method - straight line (numerical results) and dashed line (order 1). 


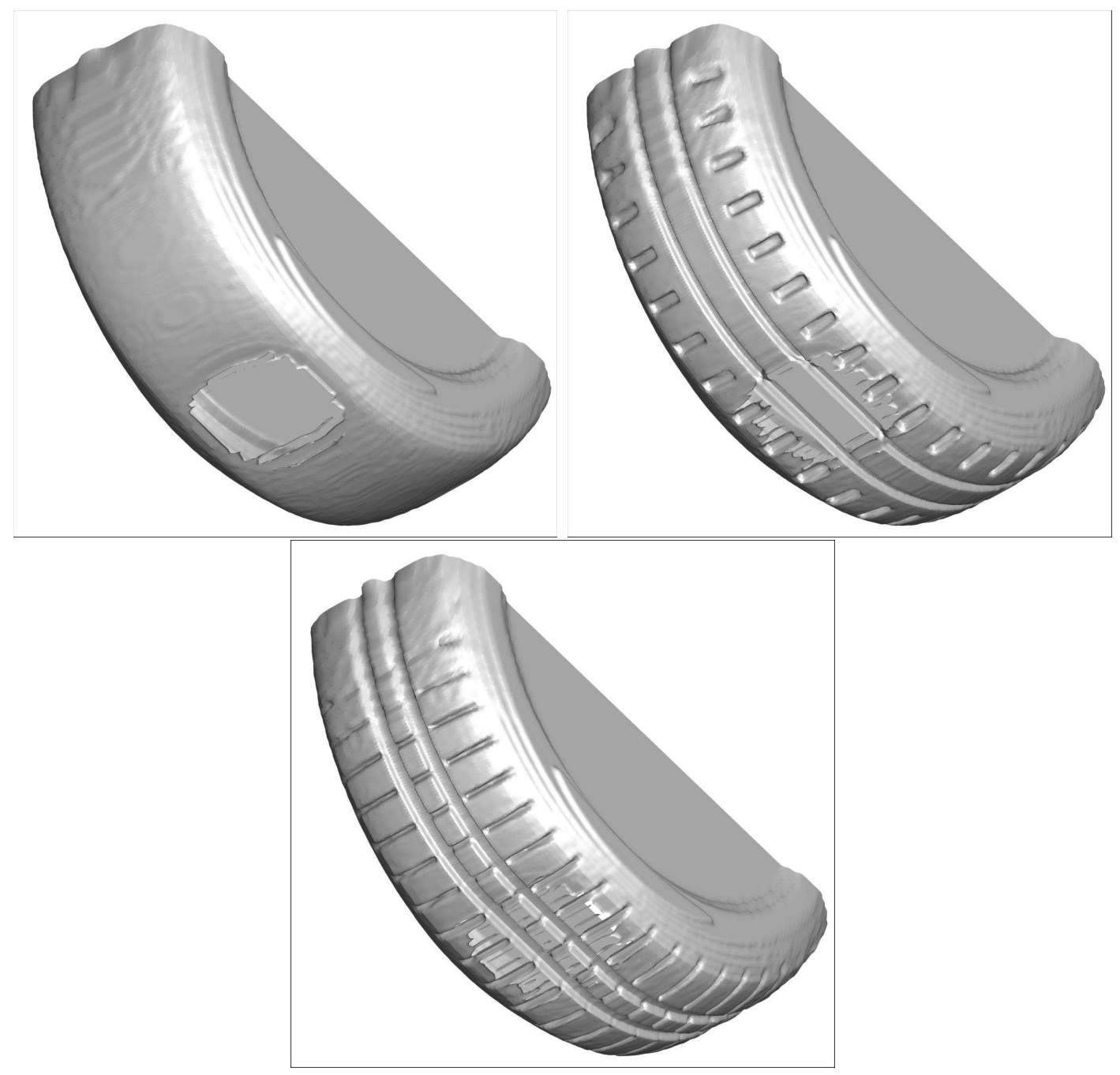

Figure 13: Topology of the three considered tires called T1, T2 and T3 (from left to right and top to bottom) - The tire patterns are provided by MICHELIN. 


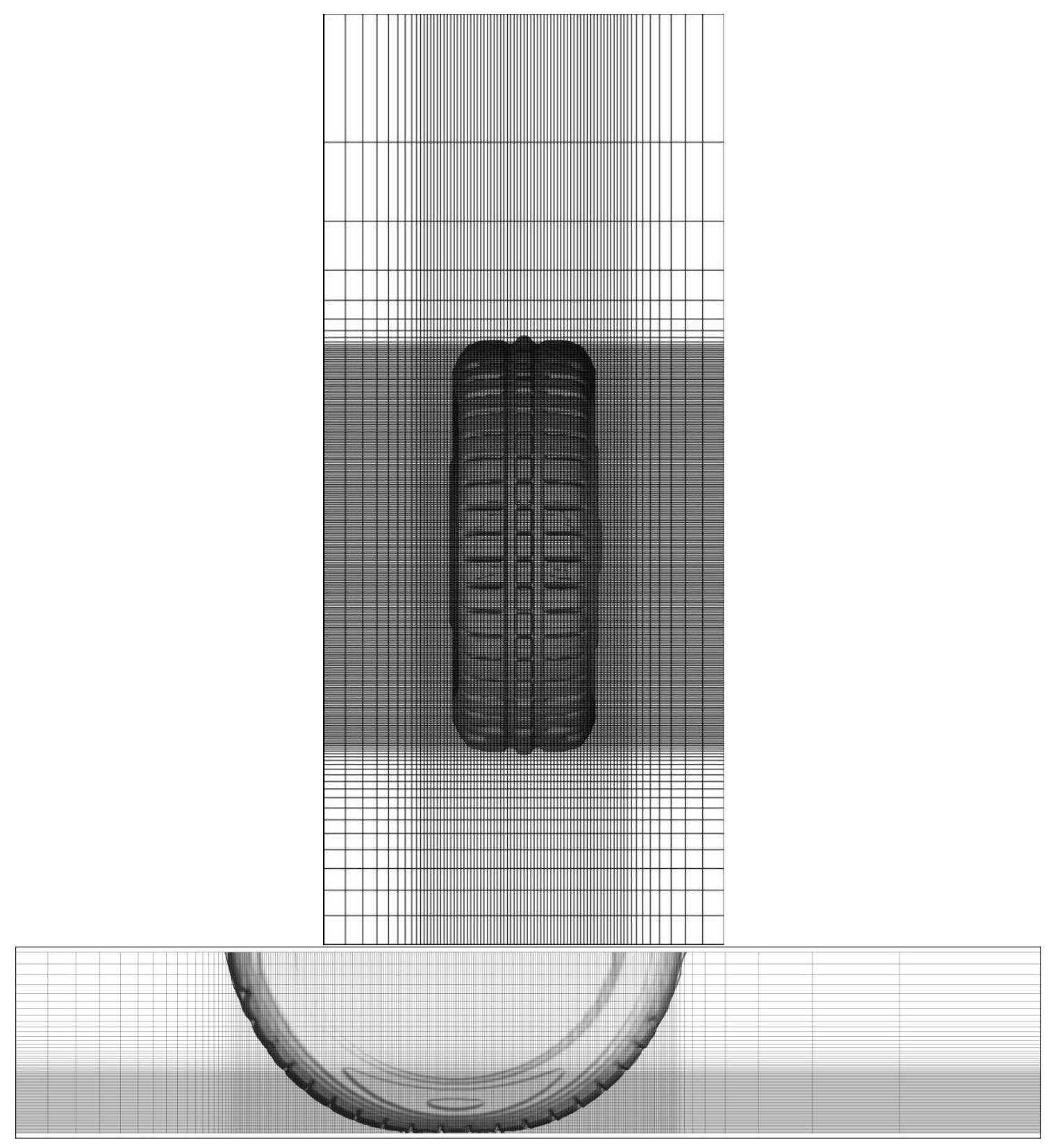

Figure 14: Structure of the calculation grid in an horizontal (top) and a vertical slice (bottom). 

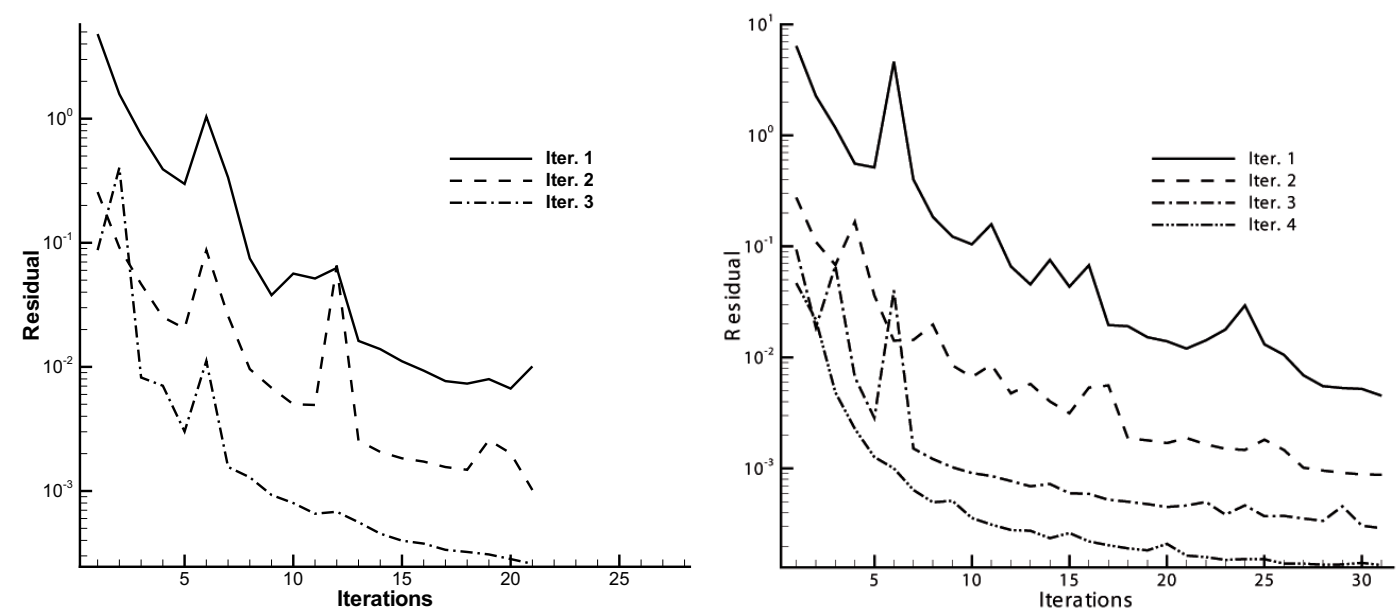

Figure 15: Convergence of the residual with $\mathcal{K}=1$ for 3 iterations of $3 \mathrm{AL}$ and 20 iterations of BiCG-Stab II (left, config. 1) and for 4 iterations of 3AL and 30 iterations of BiCG-Stab II (right, config. 2). 

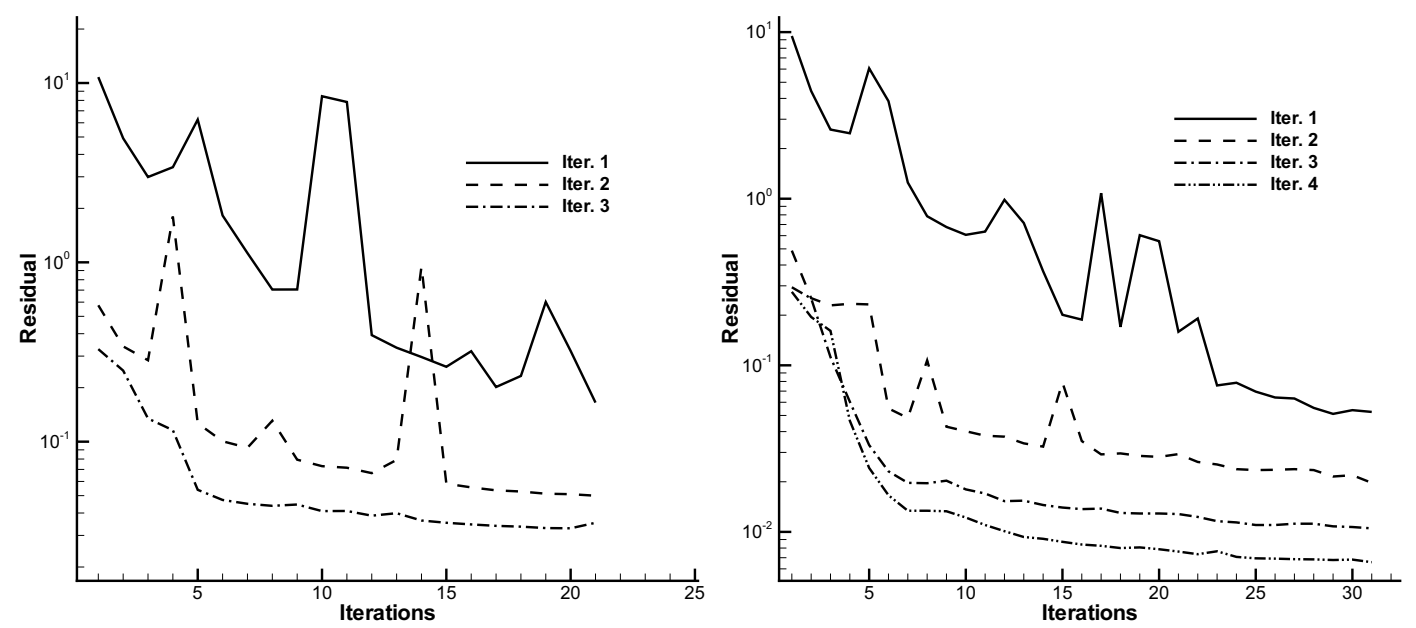

Figure 16: Convergence of the residual with $\mathcal{K}=100$ for 3 iterations of $3 \mathrm{AL}$ and 20 iterations of BiCG-Stab II (left, config. 1) and for 4 iterations of 3AL and 30 iterations of BiCG-Stab II (right, config. 2). 

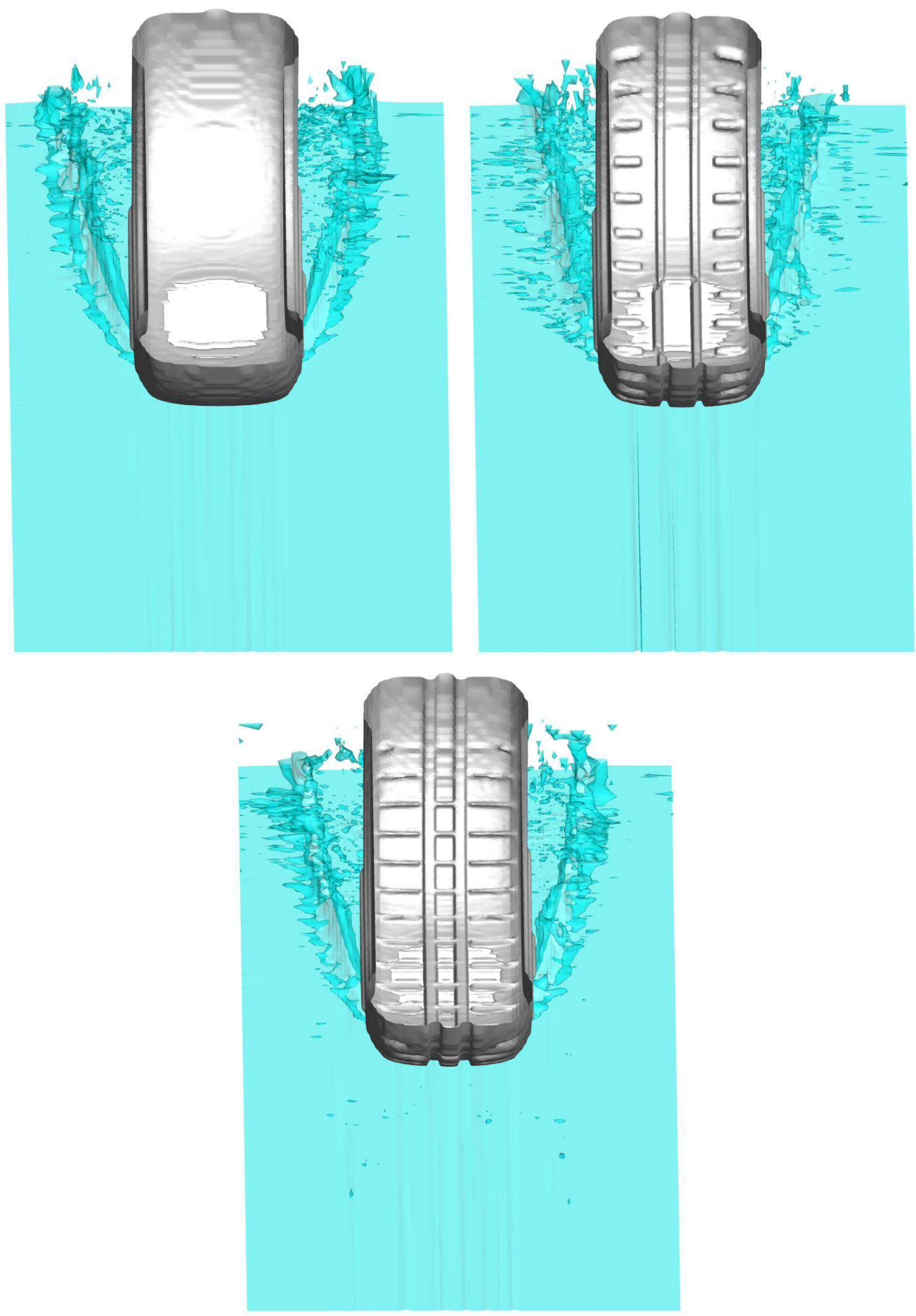

Figure 17: Two-phase flow interacting with tires T1, T2 and T3 - The tire iso-surface is plotted in grey whereas the free surface is represented in blue. 


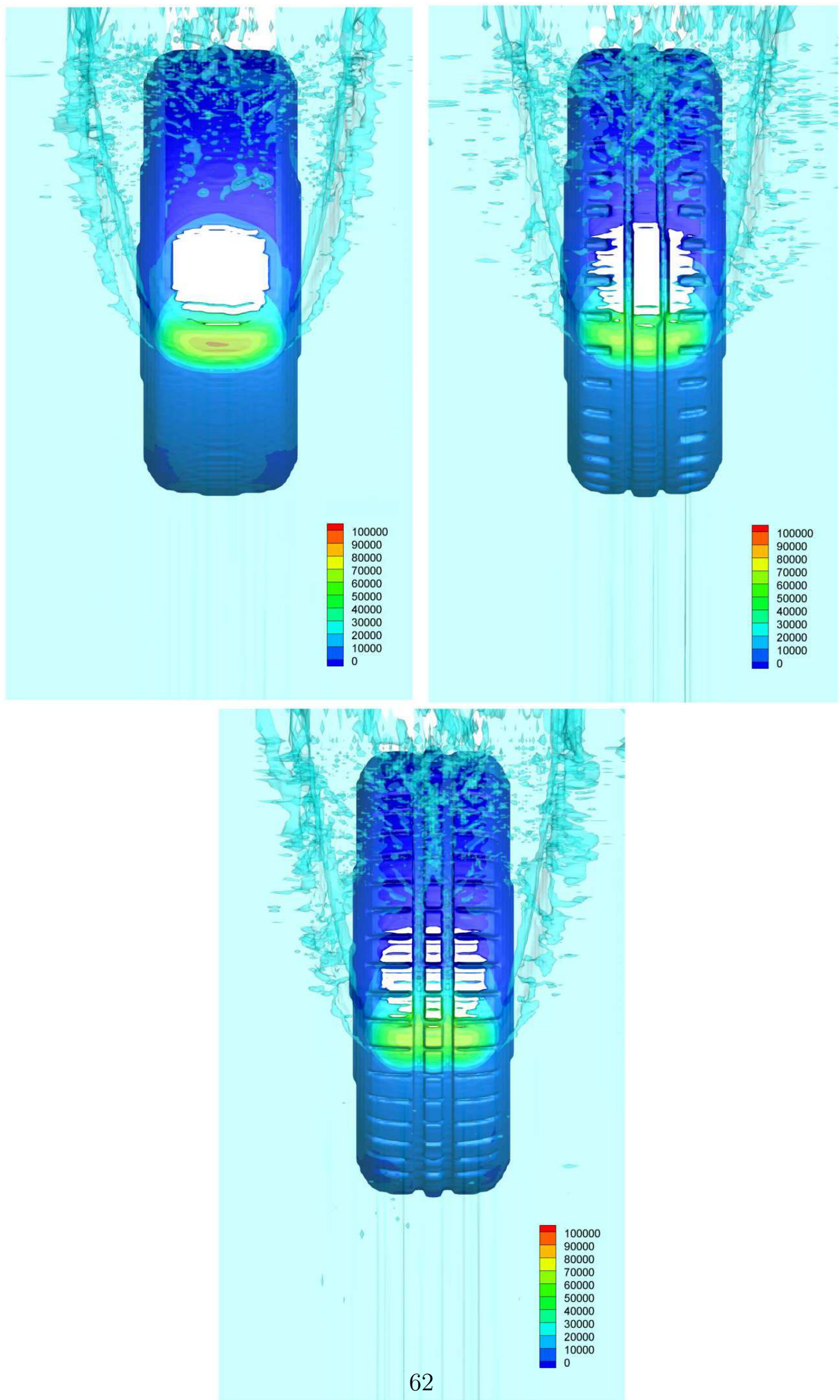

Figure 18: Two-phase flow interacting with tires $T 1, T 2$ and $T 3$ - The free surface is represented in blue with translucency whereas the iso-colors describe the pressure projected onto the tire surface. 


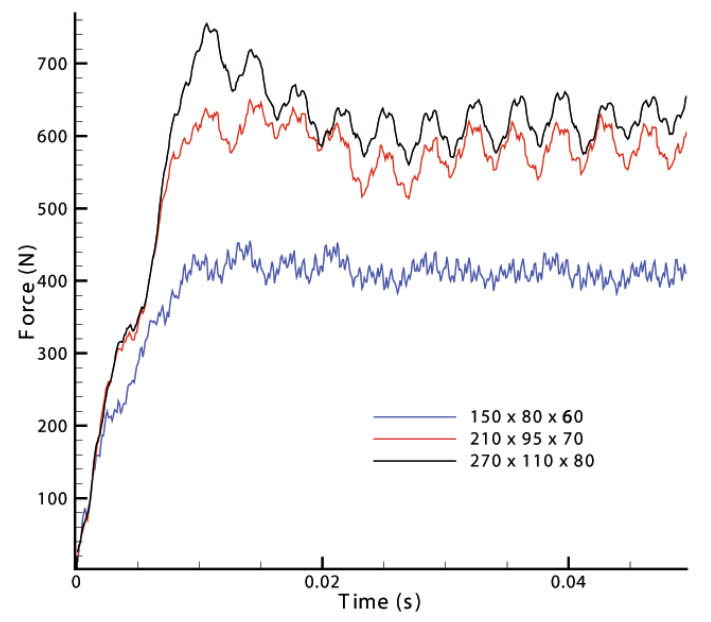

Figure 19: Time evolution of $F_{n}^{p}$ for the tire $T 2$ and three meshes. 


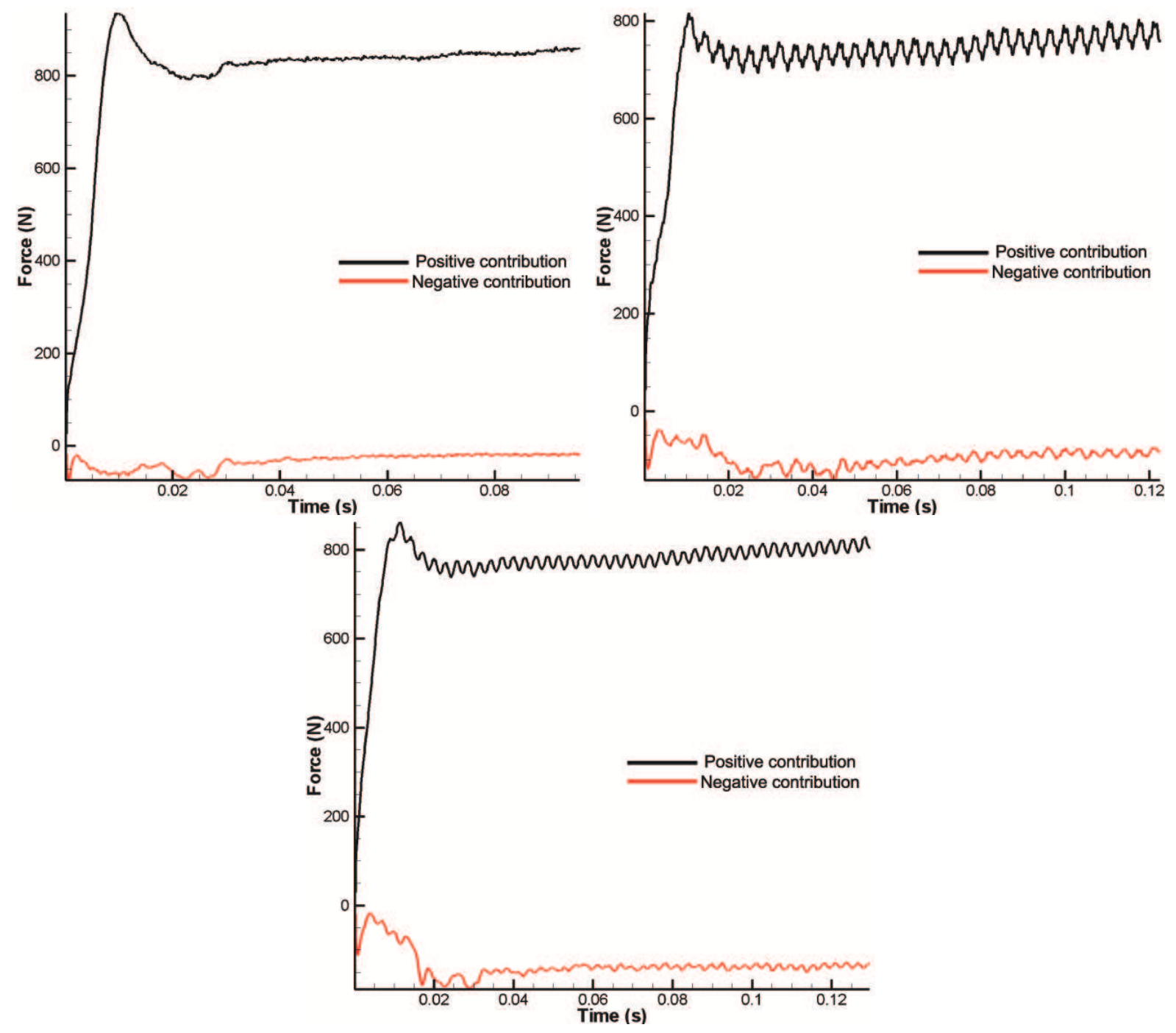

Figure 20: Time evolution of $F_{n}^{-}$and $F_{n}^{+}$for tires T1, T2 and T3 (from left to right and top to bottom). 


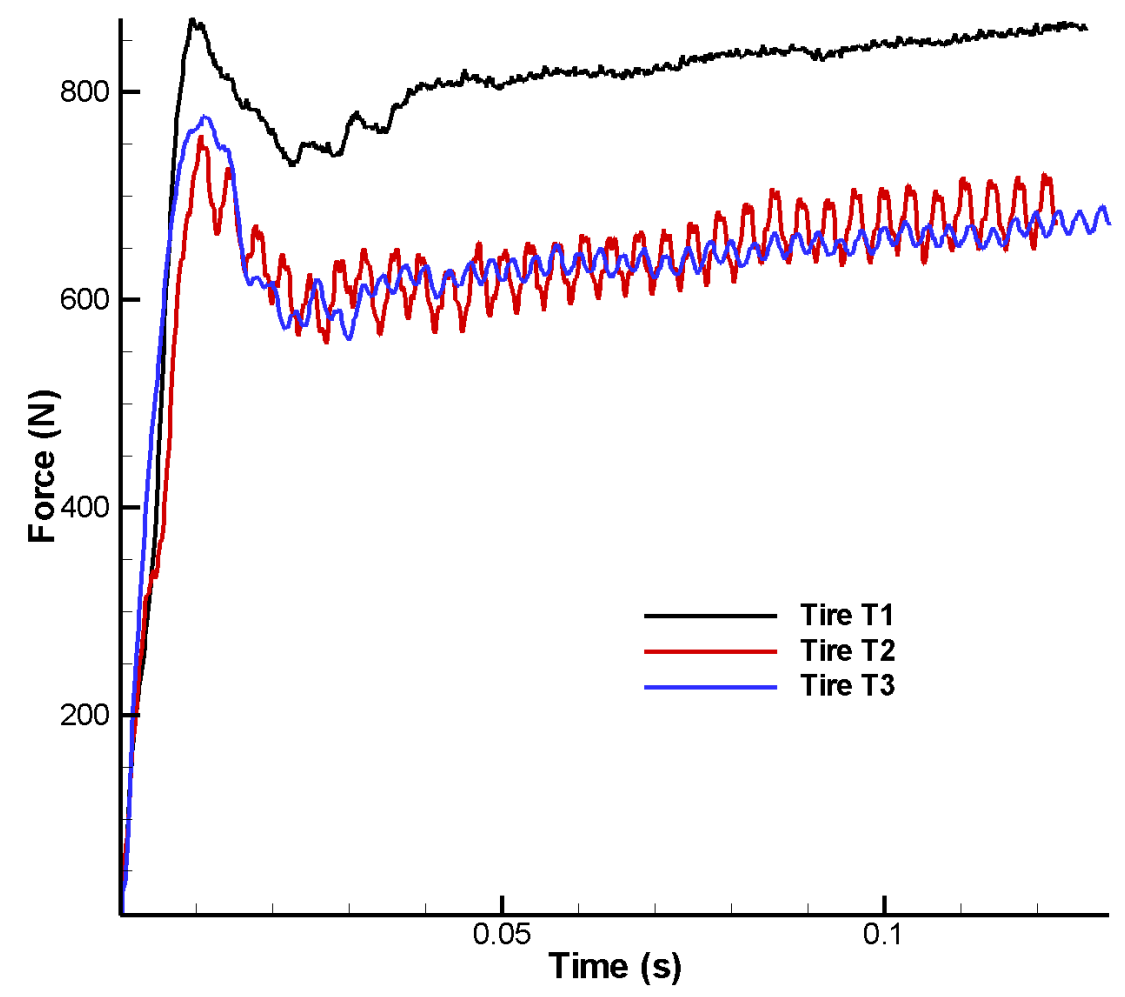

Figure 21: Time evolution of $F_{n}^{p}$ for tires $T 1, T 2$ and $T 3$. 


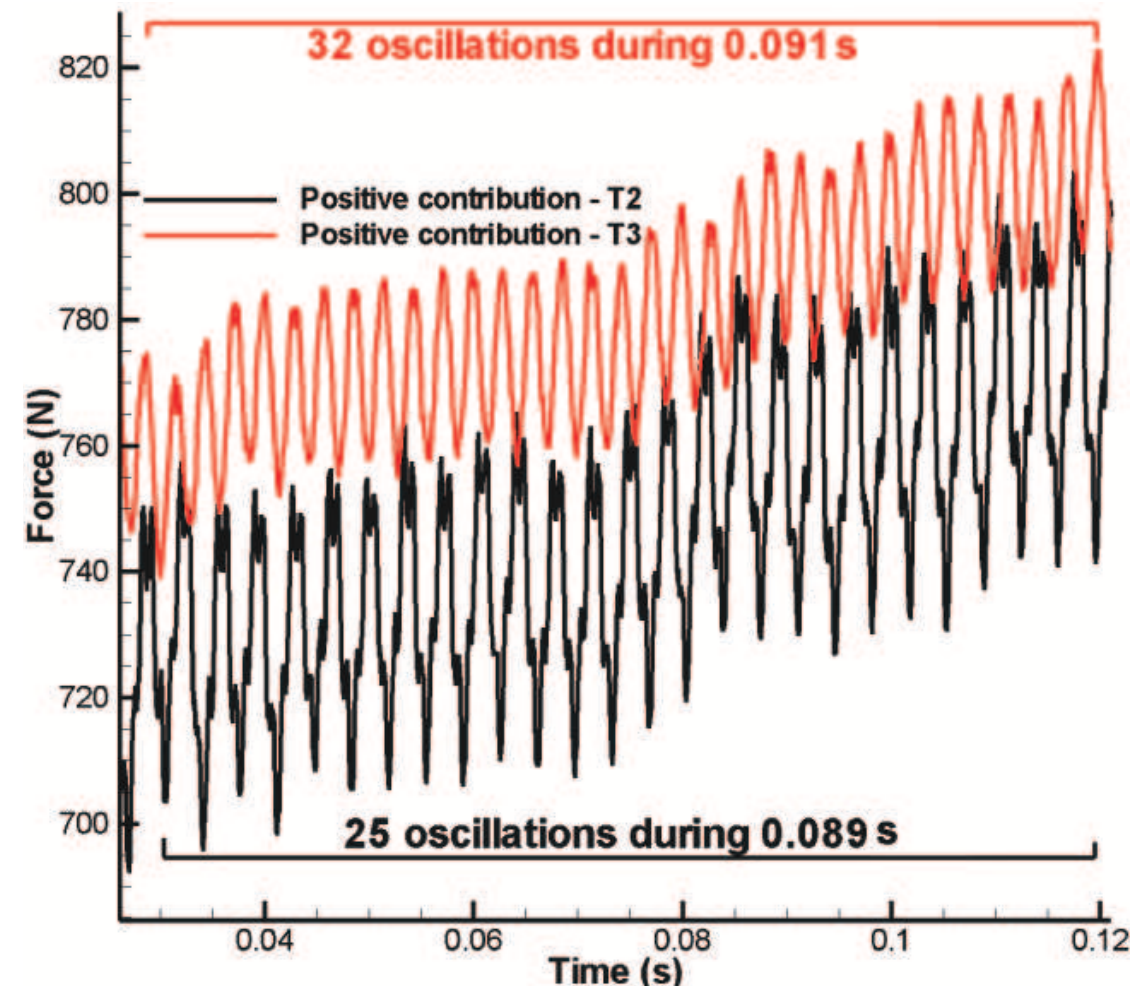

Figure 22: Zoom on the time evolution of $F_{n}^{+}$for tires $T 2$ and $T 3$. 


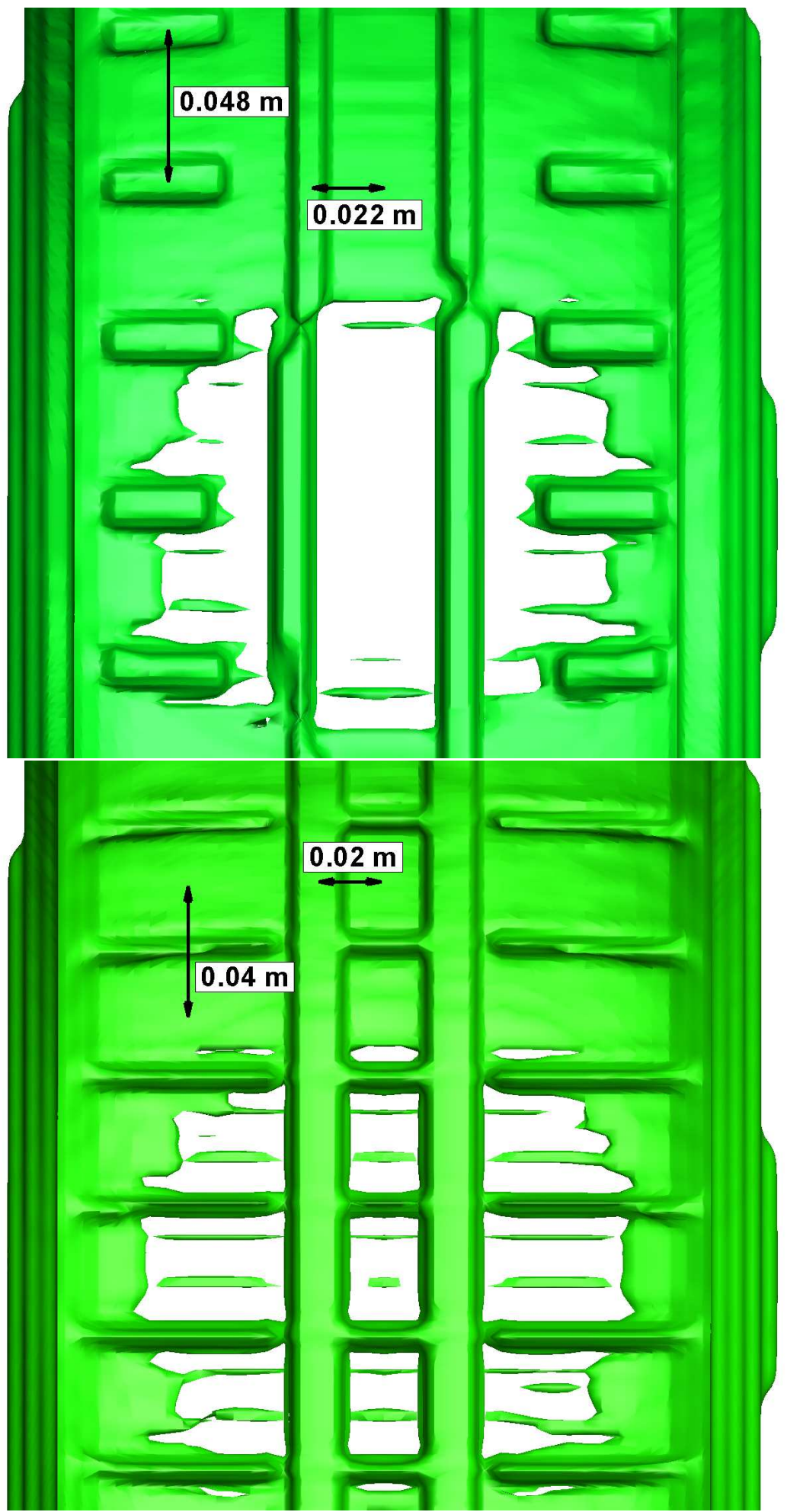

Figure 23: Typical structure of tires T2 (top) and T3 (bottom) - A downside view of the tires is presented at the contact area with the road. 